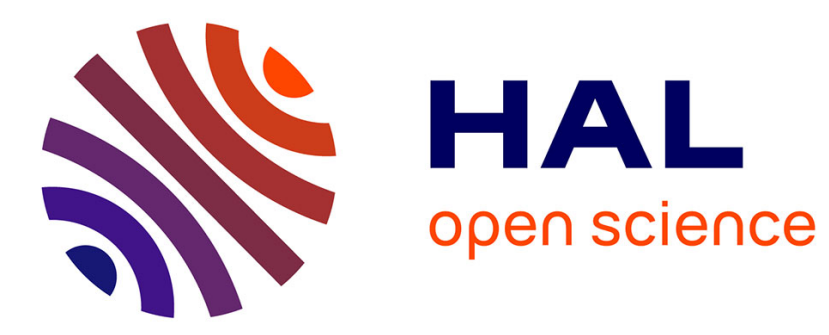

\title{
Stability results for the parameter identification inverse problem in cardiac electrophysiology
}

\author{
Jamila Lassoued, Moncef Mahjoub, Nejib Zemzemi
}

\section{To cite this version:}

Jamila Lassoued, Moncef Mahjoub, Nejib Zemzemi. Stability results for the parameter identification inverse problem in cardiac electrophysiology. Inverse Problems, 2016, 32 (11), pp.1-31. 10.1088/0266$5611 / 32 / 11 / 115002$. hal-01399373

\section{HAL Id: hal-01399373 \\ https://hal.inria.fr/hal-01399373}

Submitted on 18 Nov 2016

HAL is a multi-disciplinary open access archive for the deposit and dissemination of scientific research documents, whether they are published or not. The documents may come from teaching and research institutions in France or abroad, or from public or private research centers.
L'archive ouverte pluridisciplinaire $\mathbf{H A L}$, est destinée au dépôt et à la diffusion de documents scientifiques de niveau recherche, publiés ou non, émanant des établissements d'enseignement et de recherche français ou étrangers, des laboratoires publics ou privés. 


\title{
Stability results for the parameter identification inverse problem in cardiac electrophysiology
}

\author{
Jamila Lassoued $^{1}$, Moncef Mahjoub ${ }^{1}$ and Néjib Zemzemi ${ }^{2}$ \\ 1 Tunis El Manar University, ENIT-LAMSIN, BP 37, Le Belvédère 1002 Tunis, \\ Tunisia \\ E-mail: jamila.lassoued@enit.rnu.tn, moncef.mahjoub@lamsin.rnu.tn \\ ${ }^{2}$ INRIA, Bordeaux Sud-Ouest, 200 Avenue de la vielle Tour 33405 Talence Cedex \\ France \\ E-mail: nejib.zemzemi@inria.fr
}

\begin{abstract}
In this paper we prove a stability estimate of the parameter identification problem in cardiac electrophysiology modeling. We use the monodomain model which is a reaction diffusion parabolic equation where the reaction term is obtained by solving an ordinary differential equation. We are interested in proving the stability of the identification of the parameter $\tau_{\text {in }}$ which is the parameter that multiplies the cubic term in the reaction term. The proof of the result is based on a new Carleman-type estimate for both the PDE and ODE problems. As a consequence of the stability result we prove the uniqueness of the parameter $\tau_{\text {in }}$ giving some observations of both state variables at a given time $t_{0}$ in the whole domain and the PDE variable in a non empty open subset $w_{0}$ of the domain.
\end{abstract}

Keywords: Parameter identification, Carleman inequality, stability result , Monodomain system, Mitchell-Schaeffer model, Ionic parameters. Submitted to: Inverse Problems

\section{Introduction}

The electric wave propagation in the heart can be represented by non-linear reactiondiffusion system coupled with an ordinary differential equation called bidomain model $[16,32,33,37]$. The coupled system describes the evolution of the electrical potential $v_{m}$ in the heart. In the particular monodomain model case, the intra and extracellular conductivities have similar anisotropic ratio (See $[15,19])$. The membrane model used to describe the ionic current is Mitchell Shaeffer, was introduced by C. Mitchell and D. Schaeffer in 2003 [30]. The model consists of two ordinary differential equations, one on the transmembrane potential $v_{m}$ and an other on a gating variable $w$, (representing the opening/closing of the ion channels). This model contains four time constatant parameters $\tau_{\text {in }}, \tau_{\text {out }}, \tau_{\text {close }}$ and $\tau_{\text {open }}$. These parameters affect the shape of the action 
potential traces and consequently they affect the electrocardiograms. The sensitivity analysis of the electrocardiograms (ECGs) to the parameters of the model on the correctness of the ECGs have been investigated [5, 6, 38]. In [9], authors used a reduced order method for the identification of these parameters. In a recent work [13], we proved that in case of parameter estimation framework it is recommended to use a proper orthogonal decomposition in order to estimate $\tau_{\text {close }}, \tau_{\text {open }}$ and $\tau_{\text {out }}$. But to estimate $\tau_{\text {in }}$, the data from which the proper orthogonal decomposition basis is computed should be sufficiently rich in order to maintain a good accuracy of the results. The main reason is that the transmembrane potential is too much sensitive to this parameter. Thus stability results for the identification of the parameter $\tau_{i n}$ are very important.

In this paper, we study the stability of the inverse problem of identification of the parameter $\tau_{\text {in }}$ : the reconstruction of the coefficient $\tau_{\text {in }}$ from the measurement of solution $v_{m}$ over a space-time domain $\omega \times(0, T)$ and some measurement at fixed time $t_{0}$. The key ingredient to these stability results is a new global Carleman estimate for nonlinear coupled system with one observation.

Such problems were not considered widely in the past. However, for non linear parabolic problem, some uniqueness results were published for the one-dimensional case. In [26], the author proved a global uniqueness theorem for the $1 D$ parabolic case, using the Bukhgeim Klibanov method based on the Carleman estimates [11, 12, 23, 24, 25, 27, 28]. Kugler [29] has proved uniqueness for a $1 D$ inverse problem for a quasilinear elliptic equation. Muzylev has published a uniqueness theorem for a piecewise analytic unknown coefficient $q(u)$ in a parabolic operator [31]. Pilant and Rundell have established uniqueness under a smallness condition for an $(n D)$ forward$(1 D)$ inverse problem [34]. That is, in [34] the unknown source function $q(u)$ is a part of an $n D$ parabolic operator, and the data are given at a single point of the boundary. Klibanov and Bukhgeim [12] has proved a global uniqueness result by the method of Carleman estimates in $n$-dimensional, where the unknown coefficient $q$ depends on the solution $u$ and $(n-1)$ spatial variables. For a reaction-diffusion linear system, the Carleman estimate obtained in [2] is used to solve the inverse problem of identification of one coefficient and initial condition but the weight functions are different in the left and right hand side of their estimate. Also, in [17] a simultaneous stability result for one coefficient and for the initial conditions are obtained for a two by two reaction-diffusion linear system. In 2008, Bellassoued and al. [3] show a Carleman estimate for the Lamé system without integral term. In [18], Boulakia and Schenone show Lipschitz stability inequalities for the identification of some parameters of the Fitz Hugh-Nagumo model from measurements on the cardiac potential and the ionic variable. The main difference between our work and $[8,18]$ is that the gating variable $w$ satisfies an other ionic model for which we have a discontinuity of rate function [30] for which we will establish a new Carleman estimate.

Let $\Omega \in \mathbb{R}^{n}$ be a bounded domain of $\mathbb{R}^{n}$ with $n \leq 3$. We denote by $\nu$ the outward unit normal to $\Omega$ on $\partial \Omega$. Let $T>0$ and we consider the following monodomain model 
system:

$$
\begin{cases}\partial_{t} v_{m}-\operatorname{div}\left(\boldsymbol{\sigma} \nabla v_{m}\right)=I_{\text {app }}+I_{\text {ion }}\left(v_{m}, w, \tau_{\text {in }}, \tau_{\text {out }}\right) & \text { in } \Omega \times(0, T) \\ \partial_{t} w=g\left(v_{m}, w, \tau_{\text {close }}, \tau_{\text {open }}\right) & \text { in } \Omega \times(0, T)\end{cases}
$$

We complete this system by the initial conditions

$$
v_{m}(x, 0)=v_{0}(x) \quad \text { and } \quad w(x, 0)=w_{0}(x), \quad \forall x \in \Omega
$$

and by Neumann boundary condition on $v_{m}$

$$
\nabla v_{m} \cdot \nu=0 \quad \text { on } \quad \partial \Omega \times(0, T),
$$

where $I_{\text {app }}, I_{\text {ion }}$ and $g$ are defined by the Mitchell-Schaeffer model (see Section 2).

Our problem is to establish a stability result of the parameter identification inverse problem from the knowledge of

$$
v_{m_{\mid Q_{0}}}, \quad \nabla v_{m_{\mid Q_{0}}} \text { and } v_{m}\left(., t_{0}\right), \quad \frac{\partial^{2} v_{m}}{\partial x_{i} \partial x_{j}}\left(., t_{0}\right), \forall i, j=1 \ldots, 3, \quad w\left(., t_{0}\right)
$$

where $\omega_{0}$ be a subdomain of $\Omega, t_{0} \in(0, T)$ and $Q_{\omega_{0}}=\omega_{0} \times(0, T)$.

Our main result will be presented in Theorem 4.1, where we establish a new Global Carleman estimate for the pair of solution $\left(v_{m}, w\right)$. The uniqueness of a solution of the identifiability problem is a consequence of this main result and would be given in Corollary 4.1

The overview of the present article is as follows: Section 2, is devoted to notation and preliminary well-posedness results. In Section 3, we derive global Carleman inequality for the monodomain system with one observation. The main result is presented in Section 4. Finally, some conclusions are given in Section 5.

\section{Regularity of the solution}

In this section, we introduce the notations and basic results concerning the monodomain system coupled to the Mitchell-Schaeffer ionic model. We recall some regularity results that would be useful for proving the stability in the parameter identification problem.

\subsection{Monodomain system with Mitchell-Schaeffer model}

We consider the monodomain system that describes the propagation of the electric wave in the heart gives by

$$
\begin{cases}\partial_{t} v_{m}-\operatorname{div}\left(\boldsymbol{\sigma} \nabla v_{m}\right)=I_{a p p}+I_{i o n}\left(v_{m}, w\right) & \text { in } \Omega \times(0, T), \\ \partial_{t} w=g\left(v_{m}, w\right) & \text { in } \Omega \times(0, T), \\ \boldsymbol{\sigma} \nabla v_{m} . \nu=0 & \text { on } \Sigma, \\ v_{m}(x, 0)=v_{0}(x), w(x, 0)=w_{0}(x) & \text { for } \quad \text { a.a. } x \in \Omega,\end{cases}
$$

where $\Omega$ and $\Sigma$ denote respectively the heart domain and boundary. The time domain is given by $[0, T]$. The variable $v_{m}$, denotes the action potential and $\boldsymbol{\sigma} \stackrel{\text { def }}{=} \boldsymbol{\sigma}_{i}\left(\boldsymbol{\sigma}_{\mathrm{i}}+\boldsymbol{\sigma}_{\mathrm{e}}\right)^{-1} \boldsymbol{\sigma}_{e}$ 
is the bulk conductivity where $\boldsymbol{\sigma}_{i}$ and $\boldsymbol{\sigma}_{e}$ are the intra- and extracellular conductivity tensors. The term $I_{a p p}$ is a given source function and the ionic current $I_{i o n}$ and the function $\mathrm{g}$ depends of the considering ionic model.

In this work we use the regularized version of the Mitchell-Schaeffer cardiac cell membrane model to define the ionic current $I_{i o n}$ and the function $g$. Then the dynamics of $w$ and $I_{\text {ion }}$ are described by:

$$
\begin{gathered}
I_{\text {ion }}(v, w)=\frac{w}{\tau_{\text {in }}} v^{2}(1-v)-\frac{v}{\tau_{\text {out }}} \\
g(v, w)=\left(\frac{1}{\tau_{\text {close }}}+\frac{\tau_{\text {close }}-\tau_{\text {open }}}{\tau_{\text {close }} \tau_{\text {open }}} h_{\infty}(v)\right)\left(w-h_{\infty}(v)\right),
\end{gathered}
$$

where

$$
h_{\infty}(v)=\frac{1}{2}\left[1-\tanh \left(\frac{v-v_{\text {gate }}}{\alpha_{\text {gate }}}\right)\right]=\frac{1}{2}\left[1-\frac{1-e^{-2\left(\frac{v-v_{\text {gate }}}{\alpha_{\text {gate }}}\right)}}{1+e^{-2\left(\frac{v-v_{\text {gate }}}{\alpha_{\text {gate }}}\right)}}\right] .
$$

Here, the parameters $\alpha_{\text {gate }}, \tau_{\text {in }}<\tau_{\text {out }}<\tau_{\text {open }}, \tau_{\text {close }}$ and $0<v_{\text {gate }}<1$ are given positive constants.

\subsection{Notations and definitions}

Let $\Omega \subset \mathbb{R}^{3}$ be a bounded strongly Lipschitz domain. We denote by $L^{p}(\Omega)$ the space of functions which are in the $p$ th power integral $(1 \leq p<\infty)$, or are measurable and essentially bounded $(p=\infty)$, and by $W^{1, p}(\Omega)$ the Sobolev space of functions $\psi: \Omega \rightarrow \mathbb{R}$ which, together with their first-order weak partial derivatives, belong to the space $L^{p}(\Omega, \mathbb{R})(1 \leq p<\infty)$. For $X$ a Banach space, denote $C([0, T] ; X)$ the space of continuous functions from $[0, T]$ into $X$ equipped with the uniform convergence norm, and

$$
\|f\|_{L^{p}(0, T ; X)}=\left(\int_{0}^{T}\|f(t)\|_{X}^{p} d t\right)^{1 / p} \quad\left(=\sup _{0<t<T} e s s\|f(t)\|^{p} \quad \text { if } \quad p=\infty\right) .
$$

By definition $L^{p}(0, T ; X), p<\infty$, is the separated space of $C([0, T] ; X)$ for this norm; for $p=\infty, L^{\infty}(0, T ; X)$ is the subset of $L^{1}(0, T ; X)$ on which the $L^{\infty}$ norm is finite. It is a Banach space for $0 \leq p \leq \infty$. For $p=2$ and $X=W^{1,2}(\Omega)$, we say space of Bochner integrable mappings, see [22]. Further, we set (see [10])

$$
W^{1,2}(\Omega) /_{\mathbb{R}}=\left\{u \in W^{1,2}(\Omega), \quad \int_{\Omega} u d x=0\right\} \subset W^{1,2}(\Omega),
$$

that is a Banach space with the norm $\|u\|_{W^{1,2}(\Omega) / \mathbb{R}}=\|u\|_{W^{1,2}(\Omega)}$. We have the PoincaréWirtinger inequality

$$
\exists C>0, \quad \forall u \in W^{1,2}(\Omega) / \mathbb{R}, \quad \int_{\Omega}|u|^{2} d x \leq C \int_{\Omega}|\nabla u|^{2} d x .
$$


We denote $Q=\Omega \times[0, T], Q_{\omega_{0}}=\omega_{0} \times(0, T)$ and $\Sigma=\partial \Omega \times(0, T)$. Let $x=\left(x_{1}, x_{2}, x_{3}\right) \in \mathbb{R}^{3}$ and $t \geq 0$, respectively, as the spatial and time variables,

$$
\partial_{t}=\frac{\partial}{\partial t}, \quad \partial_{j}=\frac{\partial}{\partial x_{j}} .
$$

The gradient $\nabla$ is always taken only with respect to the spatial variables $x$. We assume that the conductivities of the intracellular and extracellular $\boldsymbol{\sigma}_{\mathrm{i}}, \boldsymbol{\sigma}_{\mathrm{e}} \in\left[L^{\infty}(\Omega)\right]^{3 \times 3}$ are symmetric and uniformly positive definite, i.e, there exist $\alpha_{i}>0$ and $\alpha_{e}>0$ such that,

$$
\xi^{T} \boldsymbol{\sigma}_{\mathrm{i}}(x) \xi \geq \alpha_{i}|\xi|^{2}, \quad \xi^{T} \boldsymbol{\sigma}_{\mathrm{e}}(x) \xi \geq \alpha_{e}|\xi|^{2}, \quad \forall \xi \in \mathbb{R}^{3} .
$$

If we do not specially state, then we always assume that $\boldsymbol{\sigma} \in\left(C^{1}(\bar{\Omega})\right)^{3 \times 3}$, and that the coefficients $\boldsymbol{\sigma}_{i j}, i, j=1,2,3$, satisfy the uniform ellipticity: there exists a constant $\mu>0$ such that

$$
\mu|\xi|^{2} \leq \xi^{T} \boldsymbol{\sigma} \xi, \quad \forall \xi \in \mathbb{R}^{3}
$$

We set

$$
|\nabla u|_{\boldsymbol{\sigma}}^{2}:=\boldsymbol{\sigma} \nabla u . \nabla u=\sum_{i, j=1}^{3} \boldsymbol{\sigma}_{i j} \partial_{j} u \partial_{i} u \quad \text { in } Q .
$$

For the monodomain system (2), we introduce the following notion of a strong and weak solutions (see [7, 38]):

Definition 2.1. A weak solution of the monodomain system (2) is a couple of functions $\left(v_{m}, w\right)$ with the regularity

$$
\begin{gathered}
v_{m} \in C^{0}\left([0, T], L^{2}(\Omega)\right) \cap L^{2}\left(0, T ; W^{1,2}(\Omega)\right) \cap L^{p}(Q) \quad \text { with } 2 \leq p \leq 6, \\
w \in W^{1,2}\left(0, T ; L^{2}(\Omega)\right) \cap C^{0}\left([0, T] ; L^{2}(\Omega)\right),
\end{gathered}
$$

and in the distributional sense satisfying, $\forall t \in[0, T]$

$$
\begin{cases}\int_{\Omega}\left(\partial_{t} v_{m}-I_{\text {ion }}\left(v_{m}, w\right)\right) \psi d x+\int_{\Omega} \nabla \psi \boldsymbol{\sigma} \nabla v_{m} d x=\int_{\Omega} I_{a p p} \psi d x & \forall \psi \in W^{1,2}(\Omega) \\ \int_{\Omega}\left(\partial_{t} w-g\left(v_{m}, w\right)\right) \psi d x=0 & \forall \psi \in L^{2}(\Omega)\end{cases}
$$

and obey, almost everywhere, the initial conditions

$$
v_{m}(x, 0)=v_{0}(x), w(x, 0)=w_{0}(x) \quad \forall x \in \Omega .
$$

In the following proposition, we determine the appropriate spaces of the solution of system (6).

Proposition 2.1. If $v_{0}$ belongs to $L^{2}(\Omega)$, Then

$$
v_{m} \in L^{\infty}\left(0, T ; L^{2}(\Omega)\right) \cap L^{2}\left(0, T ; H^{1}(\Omega)\right) \cap L^{4}\left(0, T, L^{4}(\Omega)\right) ;
$$

And if $v_{0}$ belongs to $H^{2}(\Omega)$, Then

$$
v_{m} \in W^{1, \infty}\left(0, T ; L^{2}(\Omega)\right) \cap H^{1}\left(0, T ; H^{1}(\Omega)\right) \cap L^{\infty}\left(0, T ; H^{2}(\Omega)\right) .
$$

Proof. The proof of this regularity result could be found in [18] (Proposition 3.2. page $85)$. 


\section{Global Carleman inequality for Mitchell-Schaeffer model}

In this section we establish Carleman estimate for the monodomain system coupled to the Mitchell-Scheaffer model. This Carleman estimate would be used later for the stability and uniqueness of the solution of the parameter identification problem. We are interested in identifying the parameter $\tau_{i n}$. By denoting $a=1 / \tau_{i n}$, identifying $a$ is equivalent to identifying $\tau_{i n}$.

We first have to define the weight function that we will use. This weight is fundamental in the sense that, roughly speaking, information will propagate in space along the gradient lines of this function.

\subsection{Weight functions}

Let $\omega$ be a subdomain of $\Omega$. We have the following

Lemma 3.1. Let $\omega_{0}$ be an open set such that $\overline{\omega_{0}} \subset \omega$ (for example $\omega_{0}$ can be a small open ball). Then, under the symmetric hypothesis on $\boldsymbol{\sigma}$ and (5), there exists a function $\beta \in C^{2}(\bar{\Omega})$ such that

$$
\beta(x)>0 \quad \forall x \in \Omega, \quad \beta_{\mid \partial \Omega}=0 \quad \text { and } \quad|\nabla \beta(x)|>0 \quad \forall x \in \bar{\Omega} \backslash \omega_{0} .
$$

If, moreover $\boldsymbol{\sigma} \in\left(C^{1}(\bar{\Omega})\right)^{3 \times 3}$, we have also

$$
\boldsymbol{\sigma}(x) \nabla \beta(x) . \nu(x) \leq 0, \quad x \in \partial \Omega .
$$

The proof of Lemma 3.1 is given in [21].

Remark 1. The construction of the function $\beta$ uses Morse functions and the associated approximation theorem, such a weight function is introduced in [20].

We will now use the function $\beta$ given by Lemma 3.1 to build new weight functions. Let $\lambda$ be a sufficiently large positive constant that only depends on $\Omega$ and $\omega$. For $t \in(0, T)$ we introduce the following functions:

$$
\varphi(x, t)=\frac{e^{\lambda \beta(x)}}{t(T-t)}, \quad \eta(x, t)=\frac{e^{2 \lambda\|\beta\|_{\infty}}-e^{\lambda \beta(x)}}{t(T-t)}
$$

and

$$
\bar{\varphi}(x, t)=\frac{e^{-\lambda \beta(x)}}{t(T-t)}, \quad \bar{\eta}(x, t)=\frac{e^{2 \lambda\|\beta\|_{\infty}}-e^{-\lambda \beta(x)}}{t(T-t)} .
$$

Notice that

$$
\eta(x, t) \leq \bar{\eta}(x, t) \quad \forall(x, t) \in Q .
$$

We now have for every $\lambda>0$ the following properties which will be helpful for our calculations

$$
\begin{array}{rlrl}
\nabla \varphi & =\lambda \varphi \nabla \beta, & \nabla \eta & =-\lambda \varphi \nabla \beta \\
\nabla \bar{\varphi} & =-\lambda \varphi \nabla \beta, & \nabla \bar{\eta}=\lambda \varphi \nabla \beta .
\end{array}
$$


Stability results of the monodomain system

$$
\begin{array}{rlrl}
1 \leq\left(\frac{T}{2}\right)^{2} \varphi ; & \varphi \leq\left(\frac{T}{2}\right)^{2} \varphi^{2} ; & \varphi \leq\left(\frac{T}{2}\right)^{4} \varphi^{3} \\
\left|\partial_{t} \varphi\right| & \leq T \varphi^{2} ; & \left|\partial_{t t}^{2} \varphi\right| & \leq 2 T^{2} \varphi^{3}, \\
\left|\partial_{t} \eta\right| & \leq T \varphi^{2} ; & \left|\partial_{t t}^{2} \eta\right| & \leq 2 T^{2} \varphi^{3},
\end{array}
$$

We can notice that $\eta$ tends rapidly to $+\infty$ when $t \rightarrow T$ or $t \rightarrow 0$ but that $\eta$ is uniformly bounded in $\Omega \times[\delta, T-\delta]$ if $\delta>0$. Our last weight function will depend on a second parameter $s$ and will be of the form $e^{-s \eta(x, t)}$. We can see that, for fixed $s$, this function tends very rapidly to 0 when $t \rightarrow T$ or $t \rightarrow 0$.

\subsection{Global Carleman inequality for non linear parabolic equation}

This sub-section is dedicated to prove some Carleman estimate for the solution of the monodoamain system with Mitchell-Schaeffer ionic model.

We consider the solutions $\left(v_{m}, w\right)$ and $\left(\widetilde{v}_{m}, \widetilde{w}\right)$ to the following systems

$$
\begin{cases}\partial_{t} v_{m}-\operatorname{div}\left(\boldsymbol{\sigma} \nabla v_{m}\right)=I_{a p p}+I_{\text {ion }}\left(a, v_{m}, w\right), & \text { in } Q, \\ \partial_{t} w=g\left(v_{m}, w\right), & \text { in } Q, \\ \boldsymbol{\sigma} \nabla v_{m} . \nu=0, & \text { on } \Sigma, \\ v_{m}(x, 0)=v_{m}^{0}(x), \quad w(x, 0)=w_{m}^{0}(x), & \text { in } \Omega,\end{cases}
$$

and

$$
\begin{cases}\partial_{t} \widetilde{v}_{m}-\operatorname{div}\left(\boldsymbol{\sigma} \nabla \widetilde{v}_{m}\right)=I_{a p p}+I_{\text {ion }}\left(\widetilde{a}, \widetilde{v}_{m}, \widetilde{w}\right), & \text { in } Q, \\ \partial_{t} \widetilde{w}=g\left(\widetilde{v}_{m}, \widetilde{w}\right), & \text { in } Q, \\ \boldsymbol{\sigma} \nabla \widetilde{v}_{m} \cdot \nu=0, & \text { on } \Sigma, \\ \widetilde{v}_{m}(x, 0)=\widetilde{v}_{m}^{0}(x), \quad \widetilde{w}(x, 0)=\widetilde{w}_{m}^{0}(x), & \text { in } \Omega .\end{cases}
$$

We set $V=v_{m}-\widetilde{v}_{m}, W=w-\widetilde{w}$ and $q=a-\widetilde{a}$, where $a=1 / \tau_{i n}$ and $\widetilde{a}=1 / \widetilde{\tau}_{i n}$. Then $(V, W)$ is solution to the following problem

$$
\begin{cases}\partial_{t} V-\operatorname{div}(\boldsymbol{\sigma} \nabla V)+k V^{3}=G_{1}\left(v_{m}, w, \widetilde{v}_{m}, \widetilde{w}\right) & \text { in } \Omega \times(0, T) \\ \partial_{t} W=G_{2}\left(v_{m}, w, \widetilde{v}_{m}, \widetilde{w}\right) & \text { in } \Omega \times(0, T) \\ \boldsymbol{\sigma} \nabla V \cdot \nu=0 & \text { on } \Sigma \\ V(x, 0)=V_{0}(x), \quad W(x, 0)=W_{0}(x) & \text { in } \Omega,\end{cases}
$$

here $k(x, t)=a w(x, t), G_{1}\left(v_{m}, w, \widetilde{v}_{m}, \widetilde{w}\right)=q H\left(\widetilde{v}_{m}, \widetilde{w}\right)+R\left(V, W, \widetilde{v}_{m}, \widetilde{w}\right)$, where

$$
H\left(\widetilde{v}_{m}, \widetilde{w}\right)=\widetilde{w} \widetilde{v}_{m}^{2}\left(1-\widetilde{v}_{m}\right)
$$

and

$$
\begin{aligned}
R\left(V, W, \widetilde{v}_{m}, \widetilde{w}\right) & =a \widetilde{w}\left(1-3 \widetilde{v}_{m}\right) V^{2}+a\left(\widetilde{w}_{m}\left(2-3 \widetilde{v}_{m}\right)-1 / \tau_{\text {out }}\right) V \\
& +a\left(1-3 \widetilde{v}_{m}\right) W V^{2}+a \widetilde{v}_{m}\left(2-3 \widetilde{v}_{m}\right) W V+a \widetilde{v}_{m}^{2}\left(1-\widetilde{v}_{m}\right) W .
\end{aligned}
$$

The function $G_{2}$ is given by $G_{2}\left(v_{m}, w, \widetilde{v}_{m}, \widetilde{w}\right)=g\left(v_{m}, w\right)-g\left(\widetilde{v}_{m}, \widetilde{w}\right)$,

We can now state the global Carleman inequality 
Stability results of the monodomain system

Theorem 3.1. Suppose that $\sigma$ satisfy the condition (5). Then, there exists $\lambda_{0}=$ $\lambda_{0}(\Omega, \omega) \geq 1, s_{0}=s_{0}\left(\lambda_{0}, T\right)>1$ and a positive constant $C_{0}=C_{0}(\Omega, \omega, \sigma, T)$ such that, for any $\lambda \geq \lambda_{0}$ and any $s \geq s_{0}$, the following estimate holds

$$
\begin{aligned}
&\left\|e^{-s \eta}(s \varphi)^{-1 / 2} \partial_{t} V\right\|_{2}^{2}+\left\|e^{-s \eta}(s \varphi)^{-1 / 2} \operatorname{div}(\sigma \nabla V)\right\|_{2}^{2}+s^{3} \lambda^{4}\left\|e^{-s \eta} \varphi^{3 / 2} V\right\|_{2}^{2} \\
&+s \lambda^{2}\left\|e^{-s \eta} \varphi^{1 / 2} \nabla V\right\|_{2}^{2}+s^{2} \lambda^{2}\left\|e^{-s \eta} \varphi V\right\|_{4}^{4}+\left\|e^{-s \eta} V\right\|_{6}^{6} \\
& \leq C\left(\left\|e^{-s \eta} G_{1}\left(v_{m}, w, \widetilde{v}_{m}, \widetilde{w}\right)\right\|_{2}^{2}+s^{3} \lambda^{4} \int_{Q_{\omega_{0}}} e^{-2 s \eta} \varphi^{3}|V|^{2} d x d t\right. \\
&\left.+s \lambda^{2} \int_{Q_{\omega_{0}}} e^{-2 s \eta} \varphi|\nabla V|^{2} d x d t+s^{2} \lambda^{2} \int_{Q_{\omega_{0}}} \varphi^{2} e^{-2 s \eta}|V|^{4} d x d t\right) .
\end{aligned}
$$

for any $V$ solution to the first equation of (19) and where $\|.\|_{p}, p=2,4,6$, is the $L^{p}(Q)-$ norm.

The proof of Theorem 3.1 is very much technical. It will be done by steps, following Fursikov-Imanovilov [20].

Step 1. For $s>0$, we define $\psi=e^{-s \eta} V$, we replace in equation (19) $V$ by $e^{s \eta} \psi$, and we multiply the equation by $e^{-s \eta}$, we then obtain,

$$
e^{-s \eta}\left(\partial_{t}\left(e^{s \eta} \psi\right)-\operatorname{div}\left(\boldsymbol{\sigma} \nabla\left(e^{s \eta} \psi\right)\right)+a w e^{3 s \eta} \psi^{3}\right)=e^{-s \eta} G_{1}\left(v_{m}, w, \widetilde{v}_{m}, \widetilde{w}\right)
$$

By computing (22), we obtain an equality with the following symmetric and antisymmetric operators

$$
L_{1}(\psi(x, t))+L_{2}(\psi(x, t))=F_{1}(\psi(x, t)) \quad(x, t) \in Q
$$

where

$$
\begin{aligned}
& L_{1}(\psi)=s \partial_{t} \eta \psi-s^{2} \lambda^{2} \varphi^{2}|\nabla \beta|_{\boldsymbol{\sigma}}^{2} \psi-\operatorname{div}(\boldsymbol{\sigma} \nabla \psi)+\frac{3}{4} k e^{2 s \eta} \psi^{3} \\
& L_{2}(\psi)=2 s \lambda^{2} \varphi|\nabla \beta|_{\boldsymbol{\sigma}}^{2} \psi+\partial_{t} \psi+2 s \lambda \varphi \boldsymbol{\sigma} \nabla \beta \nabla \psi+\frac{1}{4} k e^{2 s \eta} \psi^{3} \\
& F_{1}(\psi)=e^{-s \eta} G_{1}\left(v_{m}, w, \widetilde{v}_{m}, \widetilde{w}\right)-s \lambda \varphi \operatorname{div}(\boldsymbol{\sigma} \nabla \beta) \psi+s \lambda^{2} \varphi|\nabla \beta|_{\boldsymbol{\sigma}}^{2} \psi .
\end{aligned}
$$

Besides, by virtue of (9) and properties of $\eta$ we have

$$
\psi(x, 0)=\psi(x, T)=0 .
$$

Applying the $L^{2}(Q)$ norm on equation (23), we obtain

$$
\left\|L_{1}(\psi)\right\|_{2}^{2}+\left\|L_{2}(\psi)\right\|_{2}^{2}+2\left(L_{1}(\psi), L_{2}(\psi)\right)=\left\|F_{1}(\psi)\right\|_{2}^{2}
$$

where $(.,$.$) denotes the scalar product in L^{2}(Q)$.

In virtue of (24) and (25), let us compute the scalar product in the left hand side of (28), we get

$$
\left(L_{1}(\psi), L_{2}(\psi)\right)=\sum_{i, j=1}^{4} I_{i j} .
$$

In (29), all the terms $I_{i j}$ represent the scalar products of the fours terms in $L_{1}(\psi)$ by the four terms in $L_{2}(\psi)$. In the sequel, by $C$ we mean various constants independent of 
Stability results of the monodomain system

$s, \lambda$ and $T$ as we want to keep track of the powers of $s, \lambda$ and $T$ involved. In order to organize the calculations we will give particular importance to terms

$$
\begin{gathered}
J_{1}=s^{3} \lambda^{4} \int_{Q} \varphi^{3}|\psi|^{2} d x d t, J_{2}=s \lambda^{2} \int_{Q} \varphi|\nabla \psi|^{2} d x d t, J_{3}=s^{2} \lambda^{2} \int_{Q} e^{2 s \eta} \varphi^{2}|\psi|^{4} d x d t \\
\text { and } J_{4}=\int_{Q} e^{4 s \eta}|\psi|^{6} d x d t .
\end{gathered}
$$

Step 2. Now, to prove Theorem 3.1, we will start by establishing some preliminary lemmas.

Lemma 3.2. Under the same assumptions of Theorem 3.1, we have the following inquality

$$
\begin{aligned}
\left\|L_{1}(\psi)\right\|_{2}^{2}+\| & L_{2}(\psi) \|_{2}^{2}+s^{3} \lambda^{4} \int_{Q} \varphi^{3}|\nabla \beta|_{\boldsymbol{\sigma}}^{4}|\psi|^{2} d x d t+s \lambda^{2} \int_{Q} \varphi|\nabla \beta|_{\boldsymbol{\sigma}}^{2}|\nabla \psi|_{\boldsymbol{\sigma}}^{2} d x d t \\
& +s^{2} \lambda^{2} \int_{Q} \varphi^{2} k e^{2 s \eta}|\nabla \beta|_{\boldsymbol{\sigma}}^{2}|\psi|^{4} d x d t+\int_{Q} k^{2} e^{4 s \eta}|\psi|^{6} d x d t \\
\leq & C(T)\left(\left\|e^{-s \eta} G_{1}\right\|_{2}^{2}+s^{3} \lambda^{4} \int_{Q} \varphi^{3}|\psi|^{2} d x d t+s \lambda \int_{Q} \varphi|\nabla \psi|^{2} d x d t\right. \\
& \left.+s \lambda^{2} \int_{Q} e^{2 s \eta} \varphi^{2}|\psi|^{4} d x d t+\int_{Q} e^{4 s \eta}|\psi|^{6} d x d t\right)+X_{9} .
\end{aligned}
$$

where

$$
\begin{aligned}
& X_{9}=-s^{2} \lambda \int_{\Sigma} \partial_{t} \eta \varphi|\psi|^{2} \boldsymbol{\sigma} \nabla \beta . \nu d \Sigma+2 s \lambda^{2} \int_{\Sigma} \varphi|\nabla \beta|_{\boldsymbol{\sigma}}^{2} \psi \boldsymbol{\sigma} \nabla \psi \cdot \nu d \Sigma \\
&+\int_{\Sigma} \partial_{t} \psi \boldsymbol{\sigma} \nabla \psi \cdot \nu d \Sigma-s \lambda \int_{\Sigma} \varphi|\nabla \psi|_{\boldsymbol{\sigma}}^{2} \boldsymbol{\sigma} \nabla \beta . \nu d \Sigma \\
&+\frac{1}{4} \int_{\Sigma} e^{2 s \eta} k \psi^{3} \boldsymbol{\sigma} \nabla \psi \cdot \nu d \Sigma-\frac{1}{4} s \lambda \int_{\Sigma} e^{2 s \eta} k \varphi|\psi|^{4} \boldsymbol{\sigma} \nabla \beta . \nu d \Sigma \\
&+s^{3} \lambda^{3} \int_{\Sigma} \varphi^{3}|\nabla \beta|_{\boldsymbol{\sigma}}^{2}|\psi|^{2} \boldsymbol{\sigma} \nabla \beta . \nu d \Sigma+2 s \lambda \int_{\Sigma} \varphi|\boldsymbol{\sigma} \nabla \psi \cdot \nu|^{2} \boldsymbol{\sigma} \nabla \beta . \nu d \Sigma .
\end{aligned}
$$

Proof. The first term in the right hand side of equation (29)

$$
I_{11}=2 s^{2} \lambda^{2} \int_{Q} \partial_{t} \eta \varphi|\nabla \beta|_{\sigma}^{2}|\psi|^{2} d x d t .
$$

By integration by parts with respect to the time variable, the second integral $I_{12}$ can be written as follows

$$
I_{12}=s \int_{Q} \partial_{t} \eta \psi \partial_{t} \psi d x d t=-\frac{s}{2} \int_{Q} \partial_{t t} \eta|\psi|^{2} d x d t
$$

Here we have used (27) which says that $\psi(x, 0)=\psi(x, T)=0$. From (16), we obtain

$$
I_{12} \geq-C(\Omega, \omega) s T^{2} \int_{Q} \varphi^{3}|\psi|^{2} d x d t
$$


Stability results of the monodomain system

Integrating by parts the third integral, $I_{13}$, we get

$$
\begin{aligned}
I_{13}= & 2 s^{2} \lambda \int_{Q} \partial_{t} \eta \varphi \psi \boldsymbol{\sigma} \nabla \beta . \nabla \psi d x d t=s^{2} \lambda \int_{Q} \partial_{t} \eta \varphi \boldsymbol{\sigma} \nabla \beta . \nabla\left(|\psi|^{2}\right) d x d t \\
& =-s^{2} \lambda \int_{Q} \operatorname{div}\left(\partial_{t} \eta \varphi \boldsymbol{\sigma} \nabla \beta\right)|\psi|^{2} d x d t+s^{2} \lambda \int_{\Sigma} \partial_{t} \eta \varphi|\psi|^{2} \boldsymbol{\sigma} \nabla \beta . \nu d \Sigma \\
& =-s^{2} \lambda \int_{Q} \varphi \boldsymbol{\sigma} \nabla \beta \nabla \partial_{t} \eta|\psi|^{2} d x d t-s^{2} \lambda \int_{Q} \partial_{t} \eta \boldsymbol{\sigma} \nabla \beta \nabla \varphi|\psi|^{2} d x d t \\
& -s^{2} \lambda \int_{Q} \partial_{t} \eta \varphi \operatorname{div}(\boldsymbol{\sigma} \nabla \beta)|\psi|^{2} d x d t+s^{2} \lambda \int_{\Sigma} \partial_{t} \eta \varphi|\psi|^{2} \boldsymbol{\sigma} \nabla \beta . \nu d \Sigma \\
& =s^{2} \lambda^{2} \int_{Q} \varphi\left(\partial_{t} \varphi-\partial_{t} \eta\right)|\nabla \beta|_{\boldsymbol{\sigma}}^{2}|\psi|^{2} d x d t-s^{2} \lambda \int_{Q} \partial_{t} \eta \varphi d i v(\boldsymbol{\sigma} \nabla \beta)|\psi|^{2} d x d t \\
& +s^{2} \lambda \int_{\Sigma} \partial_{t} \eta \varphi|\psi|^{2} \boldsymbol{\sigma} \nabla \beta . \nu d \Sigma .
\end{aligned}
$$

Besides, due to hypothesis on $\beta$ of Lemma 3.1, we use that

$$
\begin{aligned}
& \left|\partial_{i} \boldsymbol{\sigma}_{i j} \boldsymbol{\sigma}_{k l} \partial_{k} \beta\right| \leq C(\Omega, \omega, \boldsymbol{\sigma}), \\
& \left|\boldsymbol{\sigma}_{i j} \partial_{j}\left(\boldsymbol{\sigma}_{k l} \partial_{k} \beta\right)\right| \leq C(\Omega, \omega, \boldsymbol{\sigma}), \\
& |\operatorname{div}(\boldsymbol{\sigma} \nabla \beta)| \leq C(\Omega, \omega, \boldsymbol{\sigma}), \\
& \left|\boldsymbol{\sigma}_{k l} \partial_{l} \boldsymbol{\sigma}_{i j} \partial_{k} \beta\right| \leq C(\Omega, \omega, \boldsymbol{\sigma}) \\
& \left|\boldsymbol{\sigma}_{i j} \partial_{j} \beta \partial_{i} \beta\right| \leq C(\Omega, \omega, \boldsymbol{\sigma}),
\end{aligned}
$$

then, from (15), (16), (38) and (35), we obtain

$$
I_{11}+I_{13} \geq-C(\Omega, \omega, \boldsymbol{\sigma}) s^{2} \lambda^{2} T \int_{Q} \varphi^{3}|\psi|^{2} d x d t+s^{2} \lambda \int_{\Sigma} \partial_{t} \eta \varphi|\psi|^{2} \boldsymbol{\sigma} \nabla \beta . \nu d \Sigma .
$$

We now consider the scalar product between the second term of $L_{1}(\psi)$ with the second one of $L_{2}(\psi)$. By integration by parts with respect to the time variable, the following holds,

$$
\begin{aligned}
I_{22} & =-s^{2} \lambda^{2} \int_{Q} \varphi^{2}|\nabla \beta|_{\boldsymbol{\sigma}}^{2} \psi \partial_{t} \psi d x d t=-\frac{1}{2} s^{2} \lambda^{2} \int_{Q} \varphi^{2}|\nabla \beta|_{\boldsymbol{\sigma}}^{2} \partial_{t}\left(|\psi|^{2}\right) d x d t \\
& =s^{2} \lambda^{2} \int_{Q} \varphi \partial_{t} \varphi|\nabla \beta|_{\boldsymbol{\sigma}}^{2}|\psi|^{2} d x d t
\end{aligned}
$$

Here we have used (27) which say that $\psi(x, 0)=\psi(x, T)=0$. From (15) and (40), we obtain

$$
I_{22} \geq-C(\Omega, \omega, \boldsymbol{\sigma}) s^{2} \lambda^{2} T^{2} \int_{Q} \varphi^{3}|\psi|^{2} d x d t .
$$

Integrating now by parts in the seven term of (29) we obtain,

$$
\begin{aligned}
I_{23}= & -2 s^{3} \lambda^{3} \int_{Q} \varphi^{3} \psi|\nabla \beta|_{\boldsymbol{\sigma}}^{2} \boldsymbol{\sigma} \nabla \beta \cdot \nabla \psi d x d t=-s^{3} \lambda^{3} \int_{Q} \varphi^{3}|\nabla \beta|_{\boldsymbol{\sigma}}^{2} \boldsymbol{\sigma} \nabla \beta \cdot \nabla\left(|\psi|^{2}\right) d x d t \\
& =s^{3} \lambda^{3} \int_{Q} \operatorname{div}\left(\varphi^{3}|\nabla \beta|_{\boldsymbol{\sigma}}^{2} \boldsymbol{\sigma} \nabla \beta\right)|\psi|^{2} d x d t-s^{3} \lambda^{3} \int_{\Sigma} \varphi^{3}|\nabla \beta|_{\boldsymbol{\sigma}}^{2}|\psi|^{2} \boldsymbol{\sigma} \nabla \beta . \nu d \Sigma
\end{aligned}
$$


Stability results of the monodomain system

$$
\begin{aligned}
& =3 s^{3} \lambda^{4} \int_{Q} \varphi^{3}|\nabla \beta|_{\boldsymbol{\sigma}}^{4}|\psi|^{2} d x d t+s^{3} \lambda^{3} \int_{Q} \varphi^{3} d i v\left(|\nabla \beta|_{\boldsymbol{\sigma}}^{2} \boldsymbol{\sigma} \nabla \beta\right)|\psi|^{2} d x d t \\
& -s^{3} \lambda^{3} \int_{\Sigma} \varphi^{3}|\nabla \beta|_{\boldsymbol{\sigma}}^{2}|\psi|^{2} \boldsymbol{\sigma} \nabla \beta . \nu d \Sigma .
\end{aligned}
$$

Note that $\left|\operatorname{div}\left(|\nabla \beta|_{\boldsymbol{\sigma}}^{2} \boldsymbol{\sigma} \nabla \beta\right)\right| \leq C(\Omega, \omega, \boldsymbol{\sigma})$, therefore from (44) and (40),

$$
\begin{aligned}
I_{23} \geq & 3 s^{3} \lambda^{4} \int_{Q} \varphi^{3}|\nabla \beta|_{\boldsymbol{\sigma}}^{4}|\psi|^{2} d x d t-C(\Omega, \omega, \boldsymbol{\sigma}) s^{3} \lambda^{3} \int_{Q} \varphi^{3}|\psi|^{2} d x d t \\
& -s^{3} \lambda^{3} \int_{\Sigma} \varphi^{3}|\nabla \beta|_{\boldsymbol{\sigma}}^{2}|\psi|^{2} \boldsymbol{\sigma} \nabla \beta . \nu d \Sigma .
\end{aligned}
$$

Integrating by parts the integral $I_{31}$ with respect to the space variable, we obtain

$$
\begin{aligned}
& I_{31}=-2 s \lambda^{2} \int_{Q} \varphi|\nabla \beta|_{\boldsymbol{\sigma}}^{2} \psi \operatorname{div}(\boldsymbol{\sigma} \nabla \psi) d x d t \\
&=2 s \lambda^{3} \int_{Q} \varphi|\nabla \beta|_{\boldsymbol{\sigma}}^{2} \psi \boldsymbol{\sigma} \nabla \beta \cdot \nabla \psi d x d t+2 s \lambda^{2} \int_{Q} \varphi|\nabla \beta|_{\boldsymbol{\sigma}}^{2}|\nabla \psi|_{\boldsymbol{\sigma}}^{2} d x d t \\
&+2 s \lambda^{2} \int_{Q} \varphi \psi \nabla\left(|\nabla \beta|_{\boldsymbol{\sigma}}^{2}\right) \boldsymbol{\sigma} \nabla \psi d x d t-2 s \lambda^{2} \int_{\Sigma} \varphi|\nabla \beta|_{\boldsymbol{\sigma}}^{2} \psi \boldsymbol{\sigma} \nabla \psi \cdot \nu d \Sigma .
\end{aligned}
$$

Since $\nabla \varphi=\lambda \varphi \nabla \beta$. From this and Cauchy-Schwartz inequality, we obtain

$$
\begin{aligned}
& \left.\left|2 s \lambda^{3} \int_{Q} \varphi\right| \nabla \beta\right|_{\boldsymbol{\sigma}} ^{2} \psi \boldsymbol{\sigma} \nabla \beta \cdot \nabla \psi d x d t \mid \leq \\
& 4 s \lambda^{4} \int_{Q} \varphi|\nabla \beta|_{\boldsymbol{\sigma}}^{4}|\psi|^{2} d x d t+\frac{1}{4} s \lambda^{2} \int_{Q} \varphi|\boldsymbol{\sigma} \nabla \beta|^{2}|\nabla \psi|^{2} d x d t,
\end{aligned}
$$

and

$$
\begin{aligned}
& \left|2 s \lambda^{2} \int_{Q} \varphi \psi \nabla\left(|\nabla \beta|_{\boldsymbol{\sigma}}^{2}\right) \boldsymbol{\sigma} \nabla \psi d x d t\right|=\left.\left|4 s \lambda^{2} \int_{Q} \varphi \psi\right| \nabla \beta\right|_{\boldsymbol{\sigma}} \boldsymbol{\sigma} \nabla|\nabla \beta|_{\boldsymbol{\sigma}} \cdot \nabla \psi d x d t \mid \\
& \leq\left.\left. 16 s \lambda^{2} \int_{Q} \varphi|\boldsymbol{\sigma} \nabla| \nabla \beta\right|_{\boldsymbol{\sigma}}\right|^{2}|\psi|^{2} d x d t+\frac{1}{4} s \lambda^{2} \int_{Q} \varphi|\nabla \beta|_{\boldsymbol{\sigma}}^{2}|\nabla \psi|^{2} d x d t .
\end{aligned}
$$

Since $\left.\left.|\boldsymbol{\sigma} \nabla| \nabla \beta\right|_{\boldsymbol{\sigma}}\right|^{2}<C(\Omega, \omega, \boldsymbol{\sigma})$, thus from (40), (47) and (48) we transform (46) as follows.

$$
\begin{aligned}
I_{31} \geq & \frac{3}{2} s \lambda^{2} \int_{Q} \varphi|\nabla \beta|_{\boldsymbol{\sigma}}^{2}|\nabla \psi|_{\boldsymbol{\sigma}}^{2} d x d t-C \int_{Q} s \varphi \lambda^{4}|\psi|^{2} d x d t \\
& -2 s \lambda^{2} \int_{\Sigma} \varphi|\nabla \beta|_{\boldsymbol{\sigma}}^{2} \psi \boldsymbol{\sigma} \nabla \psi \cdot \nu d \Sigma .
\end{aligned}
$$

Integrating by parts the integral $I_{32}$ with respect to the space variable, we obtain

$$
\begin{aligned}
I_{32} & =-\int_{Q} \partial_{t} \psi \operatorname{div}(\boldsymbol{\sigma} \nabla \psi) d x d t=\int_{Q} \psi \operatorname{div}\left(\boldsymbol{\sigma} \nabla \partial_{t} \psi\right) d x d t \\
& =-\int_{Q} \nabla \psi \boldsymbol{\sigma} \nabla \partial_{t} \psi d x d t+\int_{\Sigma} \psi \boldsymbol{\sigma} \nabla \partial_{t} \psi \cdot \nu d \Sigma \\
& =-\int_{\Sigma} \partial_{t} \psi \boldsymbol{\sigma} \nabla \psi \cdot \nu d \Sigma .
\end{aligned}
$$


Stability results of the monodomain system

Here we have used $\psi=e^{-s \eta} V$, and then we can write

$$
\partial_{i} \psi=\left(-s \partial_{i} \eta V+\partial_{i} V\right) e^{-s \eta}
$$

therefore

$$
\partial_{i} \psi(x, 0)=\partial_{i} \psi(x, T)=0 \quad \forall x \in \Omega, i=1,2,3 .
$$

and from $(27)$ which say that $\psi(x, 0)=\psi(x, T)=0$.

Integrating by parts the integral $I_{33}$ with respect to the space variable, we obtain

$$
\begin{gathered}
I_{33}=-2 s \lambda \int_{Q} \varphi d i v(\boldsymbol{\sigma} \nabla \psi) \boldsymbol{\sigma} \nabla \beta \cdot \nabla \psi d x d t=2 s \lambda \sum_{i, j, k, l=1}^{3} \int_{Q} \varphi \partial_{i} \boldsymbol{\sigma}_{i j} \boldsymbol{\sigma}_{k l} \partial_{k} \beta \partial_{i} \psi \partial_{l} \psi d x d t \\
\quad+2 s \lambda^{2} \int_{Q} \varphi|\boldsymbol{\sigma} \nabla \beta . \nabla \psi|^{2} d x d t-s \lambda^{2} \int_{Q} \varphi|\nabla \beta|_{\boldsymbol{\sigma}}^{2}|\nabla \psi|_{\boldsymbol{\sigma}}^{2} d x d t \\
+2 s \lambda \sum_{i, j, k, l=1}^{3} \int_{Q} \varphi \boldsymbol{\sigma}_{i j} \partial_{j}\left(\boldsymbol{\sigma} a_{k l} \partial_{k} \beta\right) \partial_{i} \psi \partial_{l} \psi d x d t-s \lambda \int_{Q} \varphi d i v(\boldsymbol{\sigma} \nabla \beta)|\nabla \psi|_{\boldsymbol{\sigma}}^{2} d x d t \\
\quad-s \lambda \sum_{i, j, k, l=1}^{3} \int_{Q} \varphi \boldsymbol{\sigma}_{k l} \partial_{l} \boldsymbol{\sigma}_{i j} \partial_{k} \beta \partial_{i} \psi \partial_{j} \psi d x d t-s \lambda \int_{\Sigma} \varphi|\nabla \psi|_{\boldsymbol{\sigma}}^{2} \boldsymbol{\sigma} \nabla \beta . \nu d \Sigma \\
=2 s \lambda^{2} \int_{Q} \varphi|\boldsymbol{\sigma} \nabla \beta . \nabla \psi|^{2} d x d t-s \lambda^{2} \int_{Q} \varphi|\nabla \beta|_{\boldsymbol{\sigma}}^{2}|\nabla \psi|_{\boldsymbol{\sigma}}^{2} d x d t+\sum_{i=1}^{4} X_{i} \\
+s \lambda \int_{\Sigma} \varphi|\nabla \psi|_{\boldsymbol{\sigma}}^{2} \boldsymbol{\sigma} \nabla \beta . \nu d \Sigma-2 s \lambda \int_{\Sigma} \varphi \boldsymbol{\sigma} \nabla \beta . \nabla \psi \boldsymbol{\sigma} \nabla \psi \cdot \nu d \Sigma,
\end{gathered}
$$

with

$$
\begin{aligned}
& X_{1}=2 s \lambda \sum_{i, j, k, l=1}^{3} \int_{Q} \varphi \partial_{i} \boldsymbol{\sigma}_{i j} \boldsymbol{\sigma}_{k l} \partial_{k} \beta \partial_{i} \psi \partial_{l} \psi d x d t \\
& X_{2}=2 s \lambda \sum_{i, j, k, l=1}^{3} \int_{Q} \varphi \boldsymbol{\sigma}_{i j} \partial_{j}\left(\boldsymbol{\sigma}_{k l} \partial_{k} \beta\right) \partial_{i} \psi \partial_{l} \psi d x d t, \\
& X_{3}=-s \lambda \int_{Q}^{\varphi} \varphi \operatorname{div}(\boldsymbol{\sigma} \nabla \beta)|\nabla \psi|_{\boldsymbol{\sigma}}^{2} d x d t
\end{aligned}
$$

and

$$
X_{4}=-s \lambda \sum_{i, j, k, l=1}^{3} \int_{Q} \varphi \boldsymbol{\sigma}_{k l} \partial_{l} \boldsymbol{\sigma}_{i j} \partial_{k} \beta \partial_{i} \psi \partial_{j} \psi d x d t .
$$

Since $\sigma$ is symmetric and $\boldsymbol{\sigma} \nabla \beta=(\boldsymbol{\sigma} \nabla \beta . \nu) \nu$, then

$$
-2 s \lambda \int_{\Sigma} \varphi \boldsymbol{\sigma} \nabla \beta . \nabla \psi \boldsymbol{\sigma} \nabla \psi \cdot \nu d \Sigma=-2 s \lambda \int_{\Sigma} \varphi|\boldsymbol{\sigma} \nabla \psi \cdot \nu|^{2} \boldsymbol{\sigma} \nabla \beta . \nu d \Sigma .
$$


Stability results of the monodomain system

Thus, using (56) in (51), we obtain, in first step, for the integral $I_{33}$, the following estimation

$$
\begin{aligned}
I_{33} \geq & \sum_{i=1}^{4} X_{i}-s \lambda^{2} \int_{Q} \varphi|\nabla \beta|_{\boldsymbol{\sigma}}^{2}|\nabla \psi|_{\boldsymbol{\sigma}}^{2} d x d t \\
& +s \lambda \int_{\Sigma} \varphi|\nabla \psi|_{\boldsymbol{\sigma}}^{2} \boldsymbol{\sigma} \nabla \beta . \nu d \Sigma-2 s \lambda \int_{\Sigma} \varphi|\boldsymbol{\sigma} \nabla \psi \cdot \nu|^{2} \boldsymbol{\sigma} \nabla \beta . \nu d \Sigma .
\end{aligned}
$$

In the second step, from (36), (37), (38), (39) and (40), we have

$$
\sum_{i=1}^{4} X_{i} \geq-C(\Omega, \omega, \boldsymbol{\sigma}) s \lambda \int_{Q} \varphi|\nabla \psi|^{2} d x d t
$$

Thus,

$$
\begin{aligned}
I_{33} \geq & -s \lambda^{2} \int_{Q} \varphi|\nabla \beta|_{\boldsymbol{\sigma}}^{2}|\nabla \psi|_{\boldsymbol{\sigma}}^{2} d x d t-C(\Omega, \omega, \boldsymbol{\sigma}) s \lambda \int_{Q} \varphi|\nabla \psi|^{2} d x d t \\
& +s \lambda \int_{\Sigma} \varphi|\nabla \psi|_{\boldsymbol{\sigma}}^{2} \boldsymbol{\sigma} \nabla \beta . \nu d \Sigma-2 s \lambda \int_{\Sigma} \varphi|\boldsymbol{\sigma} \nabla \psi \cdot \nu|^{2} \boldsymbol{\sigma} \nabla \beta . \nu d \Sigma .
\end{aligned}
$$

Applying Green's formula in $I_{34}$, we get

$$
\begin{aligned}
I_{34}= & -\frac{1}{4} \int_{Q} e^{2 s \eta} k \psi^{3} d i v(\boldsymbol{\sigma} \nabla \psi) d x d t \\
& =\frac{1}{4} \int_{Q} \nabla\left(e^{2 s \eta} k \psi^{3}\right) \boldsymbol{\sigma} \nabla \psi d x d t-\frac{1}{4} \int_{\Sigma} e^{2 s \eta} k \psi^{3} \boldsymbol{\sigma} \nabla \psi \cdot \nu d \Sigma \\
& =\frac{1}{4} \int_{Q} e^{2 s \eta}\left(2 s \nabla \eta k \psi^{3}+\nabla k \psi^{3}+3 k \psi^{2} \nabla \psi\right) \boldsymbol{\sigma} \nabla \psi d x d t-\frac{1}{4} \int_{\Sigma} e^{2 s \eta} k \psi^{3} \boldsymbol{\sigma} \nabla \psi \cdot \nu d \Sigma \\
& =-\frac{1}{2} s \lambda \int_{Q} e^{2 s \eta} k \varphi \psi^{3} \boldsymbol{\sigma} \nabla \beta . \nabla \psi d x d t+\frac{1}{4} \int_{Q} e^{2 s \eta} \psi^{3} \nabla k \boldsymbol{\sigma} \nabla \psi d x d t \\
& \quad+\frac{3}{4} \int_{Q} e^{2 s \eta} k \psi^{2}|\nabla \psi|_{\boldsymbol{\sigma}}^{2} d x d t-\frac{1}{4} \int_{\Sigma} e^{2 s \eta} k \psi^{3} \boldsymbol{\sigma} \nabla \psi \cdot \nu d \Sigma \\
= & \sum_{i=5}^{7} X_{i}-\frac{1}{4} \int_{\Sigma} e^{2 s \eta} k \psi^{3} \boldsymbol{\sigma} \nabla \psi \cdot \nu d \Sigma .
\end{aligned}
$$

With

$$
X_{5}=-\frac{1}{2} s \lambda \int_{Q} e^{2 s \eta} k \varphi \psi^{3} \boldsymbol{\sigma} \nabla \beta . \nabla \psi d x d t, \quad X_{6}=\frac{1}{4} \int_{Q} e^{2 s \eta} \nabla k \psi^{3} \boldsymbol{\sigma} \nabla \psi d x d t
$$

and

$$
X_{7}=\frac{3}{4} \int_{Q} e^{2 s \eta} k \psi^{2}|\nabla \psi|_{\sigma}^{2} d x d t
$$

Let us calculate $X_{5}$.

$$
\begin{aligned}
X_{5}= & -\frac{1}{2} s \lambda \int_{Q} e^{2 s \eta} k \varphi \psi^{3} \boldsymbol{\sigma} \nabla \beta . \nabla \psi d x d t=-\frac{1}{8} s \lambda \int_{Q} e^{2 s \eta} k \varphi \boldsymbol{\sigma} \nabla \beta . \nabla\left(|\psi|^{4}\right) d x d t \\
& =\frac{1}{8} s \lambda \int_{Q} \operatorname{div}\left(e^{2 s \eta} k \varphi \boldsymbol{\sigma} \nabla \beta\right)|\psi|^{4} d x d t-\frac{1}{8} s \lambda \int_{\Sigma} e^{2 s \eta} k \varphi|\psi|^{4} \boldsymbol{\sigma} \nabla \beta . \nu d \Sigma
\end{aligned}
$$


Stability results of the monodomain system

$$
\begin{aligned}
& =\frac{1}{8} s \lambda \int_{Q} \nabla\left(e^{2 s \eta} k \varphi\right) \boldsymbol{\sigma} \nabla \beta|\psi|^{4} d x d t+\frac{1}{8} s \lambda \int_{Q} e^{2 s \eta} k \varphi d i v(\boldsymbol{\sigma} \nabla \beta)|\psi|^{4} d x d t \\
& \quad-\frac{1}{8} s \lambda \int_{\Sigma} e^{2 s \eta} k \varphi|\psi|^{4} \boldsymbol{\sigma} \nabla \beta \cdot \nu d \Sigma \\
& =\frac{1}{8} s \lambda \int_{Q} e^{2 s \eta}(2 s \nabla \eta k \varphi+\nabla k \varphi+k \nabla \varphi) \boldsymbol{\sigma} \nabla \beta|\psi|^{4} d x d t \\
& +\frac{1}{8} s \lambda \int_{Q} e^{2 s \eta} k \varphi d i v(\boldsymbol{\sigma} \nabla \beta)|\psi|^{4} d x d t-\frac{1}{8} s \lambda \int_{\Sigma} e^{2 s \eta} k \varphi|\psi|^{4} \boldsymbol{\sigma} \nabla \beta . \nu d \Sigma \\
& =\frac{1}{8} s \lambda \int_{Q} e^{2 s \eta}\left(-2 s \lambda \nabla \beta k \varphi^{2}+\nabla k \varphi+k \lambda \nabla \beta \varphi\right) \boldsymbol{\sigma} \nabla \beta|\psi|^{4} d x d t \\
& +\frac{1}{8} s \lambda \int_{Q} e^{2 s \eta} k \varphi d i v(\boldsymbol{\sigma} \nabla \beta)|\psi|^{4} d x d t-\frac{1}{8} s \lambda \int_{\Sigma} e^{2 s \eta} k \varphi|\psi|^{4} \boldsymbol{\sigma} \nabla \beta . \nu d \Sigma \\
& =-\frac{1}{4} s^{2} \lambda^{2} \int_{Q} e^{2 s \eta}|\nabla \beta|_{\boldsymbol{\sigma}}^{2} k \varphi^{2}|\psi|^{4} d x d t+\frac{1}{8} s \lambda \int_{Q} e^{2 s \eta} \varphi \boldsymbol{\sigma} \nabla \beta . \nabla k|\psi|^{4} d x d t \\
& +\frac{1}{8} s \lambda^{2} \int_{Q} e^{2 s \eta} k|\nabla \beta|_{\boldsymbol{\sigma}}^{2} \varphi|\psi|^{4} d x d t-\frac{1}{8} s \lambda \int_{\Sigma} e^{2 s \eta} k \varphi|\psi|^{4} \boldsymbol{\sigma} \nabla \beta . \nu d \Sigma \\
& \quad+\frac{1}{8} s \lambda \int_{Q} e^{2 s \eta} k \varphi d i v(\boldsymbol{\sigma} \nabla \beta)|\psi|^{4} d x d t \\
& =-\frac{1}{4} s^{2} \lambda^{2} \int_{Q} e^{2 s \eta}|\nabla \beta|_{\boldsymbol{\sigma}}^{2} k \varphi^{2}|\psi|^{4} d x d t-\frac{1}{8} s \lambda \int_{\Sigma} e^{2 s \eta} k \varphi|\psi|^{4} \boldsymbol{\sigma} \nabla \beta . \nu d \Sigma+X_{8},
\end{aligned}
$$

with

$$
\begin{gathered}
X_{8}=\frac{1}{8} s \lambda \int_{Q} e^{2 s \eta} \varphi \boldsymbol{\sigma} \nabla \beta . \nabla k|\psi|^{4} d x d t+\frac{1}{8} s \lambda^{2} \int_{Q} e^{2 s \eta} k|\nabla \beta|_{\boldsymbol{\sigma}}^{2} \varphi|\psi|^{4} d x d t \\
+\frac{1}{8} s \lambda \int_{Q} e^{2 s \eta} k \varphi \operatorname{div}(\boldsymbol{\sigma} \nabla \beta)|\psi|^{4} d x d t .
\end{gathered}
$$

Thus,

$$
\begin{aligned}
I_{34}=-\frac{1}{4} s^{2} \lambda^{2} & \int_{Q} e^{2 s \eta}|\nabla \beta|_{\boldsymbol{\sigma}}^{2} k \varphi^{2}|\psi|^{4} d x d t+X_{6}+X_{7}+X_{8} \\
& -\frac{1}{4} \int_{\Sigma} e^{2 s \eta} k \psi^{3} \boldsymbol{\sigma} \nabla \psi \cdot \nu d \Sigma-\frac{1}{8} s \lambda \int_{\Sigma} e^{2 s \eta} k \varphi|\psi|^{4} \boldsymbol{\sigma} \nabla \beta . \nu d \Sigma .
\end{aligned}
$$

On the other hand, observing that

$$
I_{43}=-3 X_{5},
$$

then, inserting the above equality (64) in the integral $I_{34}$, it yields

$$
I_{34}+I_{43}=-2 X_{5}+X_{6}+X_{7}-\frac{1}{4} \int_{\Sigma} e^{2 s \eta} k \psi^{3} \boldsymbol{\sigma} \nabla \psi \cdot \nu d \Sigma .
$$

Now, we are working with $I_{34}+I_{43}$. In fact,

$$
\left|X_{8}\right| \leq C s \lambda^{2} T^{2} \int_{Q} e^{2 s \eta} \varphi^{2}|\psi|^{4} d x d t .
$$


Stability results of the monodomain system

Therefore by (61), we obtain

$$
\begin{gathered}
-2 X_{5} \geq \frac{1}{2} s^{2} \lambda^{2} \int_{Q} e^{2 s \eta} \varphi^{2} k|\nabla \beta|_{\boldsymbol{\sigma}}^{2}|\psi|^{4} d x d t-C s \lambda^{2} T^{2} \int_{Q} e^{2 s \eta} \varphi^{2}|\psi|^{4} d x d t \\
+\frac{1}{4} s \lambda \int_{\Sigma} e^{2 s \eta} k \varphi \psi^{4} \boldsymbol{\sigma} \nabla \beta . \nu d \Sigma .
\end{gathered}
$$

Using Young inequality $a b \leq \varepsilon a^{2}+C_{\varepsilon} b^{2}, \varepsilon \ll 1$, one could check that

$$
\begin{aligned}
\left|X_{6}\right| & \leq \frac{1}{4} \int_{Q} e^{2 s \eta}|\nabla k||\psi|^{3}|\sigma \nabla \psi| d x d t \\
& \leq \frac{1}{4}\left(\varepsilon \int_{Q} e^{4 s \eta}|\nabla k|^{2}|\psi|^{6} d x d t+C_{\varepsilon} \int_{Q}|\sigma \nabla \psi|^{2} d x d t\right) \\
& \leq C\left(\varepsilon \int_{Q} e^{4 s \eta}|\psi|^{6} d x d t+C_{\varepsilon} T^{2} \int_{Q} \varphi|\nabla \psi|^{2} d x d t\right) .
\end{aligned}
$$

Here we have used that $k \in L^{\infty}(Q)$ and $\boldsymbol{\sigma} \in\left[L^{\infty}(\Omega)\right]^{3 \times 3}$. Using (60), (64), (65), (61) and (68), we obtain

$$
\begin{aligned}
I_{34}+I_{43} \geq \frac{1}{2} s^{2} \lambda^{2} \int_{Q} e^{2 s \eta} \varphi^{2} k|\nabla \beta|_{\boldsymbol{\sigma}}^{2}|\psi|^{4} d x d t-C s \lambda^{2} T^{2} \int_{Q} e^{2 s \eta} \varphi^{2}|\psi|^{4} d x d t \\
-C\left(\varepsilon \int_{Q} e^{4 s \eta}|\psi|^{6} d x d t+C_{\varepsilon} T^{2} \int_{Q} \varphi|\nabla \psi|^{2} d x d t\right) \\
-\frac{1}{4} \int_{\Sigma} e^{2 s \eta} k \psi^{3} \boldsymbol{\sigma} \nabla \psi \cdot \nu d \Sigma+\frac{1}{4} s \lambda \int_{\Sigma} e^{2 s \eta} k \varphi|\psi|^{4} \boldsymbol{\sigma} \nabla \beta . \nu d \Sigma .
\end{aligned}
$$

Here we have used $X_{7} \geq 0$ and $k=a w \geq 0(0<w<1)$.

By integrating by parts the term $I_{42}$ with respect to the time variable and using the fact that $0<w<1$ (see [7] Lemma 3.3) and a is positive, we have

$$
\begin{aligned}
I_{42} & =\frac{3}{4} \int_{Q} e^{2 s \eta} k \psi^{3} \partial_{t} \psi d x d t=-\frac{3}{16} \int_{Q} e^{2 s \eta}\left(\partial_{t} k+2 s \partial_{t} \eta k\right)|\psi|^{4} d x d t \\
& \geq-C(1+s T) \int_{Q} e^{2 s \eta} \varphi^{2}|\psi|^{4} d x d t .
\end{aligned}
$$

Using (16), (40) and the fact $k \in L^{\infty}(Q)$, we have

$$
I_{14}=\frac{1}{4} s \int_{Q} \partial_{t} \eta k e^{2 s \eta}|\psi|^{4} d x d t \geq-C s T \int_{Q} e^{2 s \eta} \varphi^{2}|\psi|^{4} d x d t .
$$

and

$$
I_{41}=\frac{3}{2} s \lambda^{2} \int_{Q} k e^{2 s \eta} \varphi|\nabla \beta|_{\boldsymbol{\sigma}}^{2}|\psi|^{4} d x d t \geq-C s \lambda^{2} T^{2} \int_{Q} e^{2 s \eta} \varphi^{2}|\psi|^{4} d x d t .
$$

Finally, we have

$I_{21}=-2 s^{3} \lambda^{4} \int_{Q} \varphi^{3}|\nabla \beta|_{\boldsymbol{\sigma}}^{4}|\psi|^{2} d x d t, \quad I_{24}=-\frac{1}{4} s^{2} \lambda^{2} \int_{Q} \varphi^{2} k e^{2 s \eta}|\nabla \beta|_{\boldsymbol{\sigma}}^{2}|\psi|^{4} d x d t$

and

$$
I_{44}=\frac{3}{16} \int_{Q} k^{2} e^{4 s \eta}|\psi|^{6} d x d t
$$


Stability results of the monodomain system

Making use of estimations (34), (41), (43), (45), (49), (50), (59), (65), (70), (71), (73), (72) and (74) we obtain the lower bound for $2\left(L_{1}(\psi), L_{2}(\psi)\right)$, we get the following desired inequality:

$$
\begin{aligned}
\left\|L_{1}(\psi)\right\|^{2}+\| & L_{2}(\psi) \|^{2}+s^{3} \lambda^{4} \int_{Q} \varphi^{3}|\nabla \beta|_{\boldsymbol{\sigma}}^{4}|\psi|^{2} d x d t+s \lambda^{2} \int_{Q} \varphi|\nabla \beta|_{\boldsymbol{\sigma}}^{2}|\nabla \psi|_{\boldsymbol{\sigma}}^{2} d x d t \\
& +s^{2} \lambda^{2} \int_{Q} \varphi^{2} k e^{2 s \eta}|\nabla \beta|_{\boldsymbol{\sigma}}^{2}|\psi|^{4} d x d t+\int_{Q} k^{2} e^{4 s \eta}|\psi|^{6} d x d t \\
& \leq\left\|F_{1}\right\|_{2}^{2}+C(T)\left(s^{3} \lambda^{4} \int_{Q} \varphi^{3}|\psi|^{2} d x d t+s \lambda \int_{Q} \varphi|\nabla \psi|^{2} d x d t\right. \\
& \left.+s \lambda^{2} \int_{Q} e^{2 s \eta} \varphi^{2}|\psi|^{4} d x d t+\int_{Q} e^{4 s \eta}|\psi|^{6} d x d t\right)+X_{9}
\end{aligned}
$$

where

$$
\begin{aligned}
X_{9}=-s^{2} & \lambda \int_{\Sigma} \partial_{t} \eta \varphi|\psi|^{2} \boldsymbol{\sigma} \nabla \beta . \nu d \Sigma+2 s \lambda^{2} \int_{\Sigma} \varphi|\nabla \beta|_{\boldsymbol{\sigma}}^{2} \psi \boldsymbol{\sigma} \nabla \psi \cdot \nu d \Sigma \\
& +\int_{\Sigma} \partial_{t} \psi \boldsymbol{\sigma} \nabla \psi \cdot \nu d \Sigma-s \lambda \int_{\Sigma} \varphi|\nabla \psi|_{\boldsymbol{\sigma}}^{2} \boldsymbol{\sigma} \nabla \beta . \nu d \Sigma \\
& +\frac{1}{4} \int_{\Sigma} e^{2 s \eta} k \psi \psi^{3} \boldsymbol{\sigma} \nabla \psi \cdot \nu d \Sigma-\frac{1}{4} s \lambda \int_{\Sigma} e^{2 s \eta} k \varphi|\psi|^{4} \boldsymbol{\sigma} \nabla \beta . \nu d \Sigma \\
& +s^{3} \lambda^{3} \int_{\Sigma} \varphi^{3}|\nabla \beta|_{\boldsymbol{\sigma}}^{2}|\psi|^{2} \boldsymbol{\sigma} \nabla \beta . \nu d \Sigma+2 s \lambda \int_{\Sigma} \varphi|\boldsymbol{\sigma} \nabla \psi \cdot \nu|^{2} \boldsymbol{\sigma} \nabla \beta . \nu d \Sigma,
\end{aligned}
$$

and $F_{1}$ is given by (26). Here we used the obvious inequalities $\varphi^{-1} \leq(T / 2)^{2}$, $\varphi^{-2} \leq(T / 2)^{4}$ and the fact that the parameters $s>1, \lambda>1$.

In order to complete the estimations involving the terms of (28), we need to obtain the upper bounds for the $L^{2}$ integrals of $F_{1}(\psi)$. From the equation $(26)$, we see that

$$
\left\|F_{1}(\psi)\right\|_{2}^{2} \leq\left\|e^{-s \eta} G_{1}\right\|_{2}^{2}+C\left(s \lambda T^{2}+s \lambda^{2} T^{2}\right) \int_{Q} \varphi^{3}|\psi|^{2} d x d t .
$$

Using the inequalities, (75) and (77) we prove Lemma 3.2.

Step 3. In this step, we aim at eliminating the boundary integrals in $X_{9}$. By defining $\bar{\psi}=e^{-s \bar{\eta}} V$, replacing $V$ by $e^{s \bar{\eta}} \bar{\psi}$ in equation (19) and by multiplying the equation by $e^{-s \bar{\eta}}$, we obtain

$$
\bar{L}_{1}(\bar{\psi}(x, t))+\bar{L}_{2}(\bar{\psi}(x, t))=\bar{F}_{1}(\bar{\psi}(x, t)) \quad \text { in } \quad(x, t) \in Q
$$

where

$$
\begin{aligned}
& \bar{L}_{1}(\bar{\psi})=s \partial_{t} \bar{\eta} \bar{\psi}-s^{2} \lambda^{2} \bar{\varphi}^{2}|\nabla \beta|_{\boldsymbol{\sigma}}^{2} \bar{\psi}-\operatorname{div}(\boldsymbol{\sigma} \nabla \bar{\psi})+\frac{3}{4} k e^{2 s \bar{\eta}} \bar{\psi}^{3} \\
& \bar{L}_{2}(\bar{\psi})=2 s \lambda^{2} \bar{\varphi}|\nabla \beta|_{\boldsymbol{\sigma}}^{2} \bar{\psi}+\partial_{t} \bar{\psi}-2 s \lambda \bar{\varphi} \boldsymbol{\sigma} \nabla \beta \nabla \bar{\psi}+\frac{1}{4} k e^{2 s \bar{\eta}} \bar{\psi}^{3} \\
& \bar{F}_{1}(\bar{\psi})=e^{-s \bar{\eta}} G_{1}+s \lambda \bar{\varphi} \operatorname{div}(\boldsymbol{\sigma} \nabla \beta) \bar{\psi}+s \lambda^{2} \bar{\varphi}|\nabla \beta|_{\boldsymbol{\sigma}}^{2} \bar{\psi}
\end{aligned}
$$

In this step we prove the following lemma 
Stability results of the monodomain system

Lemma 3.3. Under the same assumptions of Theorem 3.1, we have the following inquality

$$
\begin{aligned}
\left\|L_{1}(\psi)\right\|^{2}+\| & L_{2}(\psi) \|^{2}+s^{3} \lambda^{4} \int_{Q} \varphi^{3}|\nabla \beta|_{\boldsymbol{\sigma}}^{4}|\psi|^{2} d x d t+s \lambda^{2} \int_{Q} \varphi|\nabla \beta|_{\boldsymbol{\sigma}}^{2}|\nabla \psi|_{\boldsymbol{\sigma}}^{2} d x d t \\
& +s^{2} \lambda^{2} \int_{Q} \varphi^{2} k e^{2 s \eta}|\nabla \beta|_{\boldsymbol{\sigma}}^{2}|\psi|^{4} d x d t+\int_{Q} k^{2} e^{4 s \eta}|\psi|^{6} d x d t \\
& \leq 2\left\|e^{-s \eta} G_{1}\right\|_{2}^{2}+C(T)\left(s^{3} \lambda^{4} \int_{Q} \varphi^{3}|\psi|^{2} d x d t+s \lambda \int_{Q} \varphi|\nabla \psi|^{2} d x d t\right. \\
& \left.+s \lambda^{2} \int_{Q} e^{2 s \eta} \varphi^{2}|\psi|^{4} d x d t+\int_{Q} e^{4 s \eta}|\psi|^{6} d x d t\right) .
\end{aligned}
$$

Proof. Using the same procedure as for the proof of Lemma 3.2, but here for equations (78)-(81), we obtain the following inequality

$$
\begin{aligned}
\left\|\bar{L}_{1}(\bar{\psi})\right\|^{2}+\left\|\bar{L}_{2}(\bar{\psi})\right\|^{2}+s^{3} \lambda^{4} \int_{Q} \bar{\varphi}^{3}|\nabla \beta|_{\boldsymbol{\sigma}}^{4}|\bar{\psi}|^{2} d x d t+s \lambda^{2} \int_{Q} \bar{\varphi}|\nabla \beta|_{\boldsymbol{\sigma}}^{2}|\nabla \bar{\psi}|_{\boldsymbol{\sigma}}^{2} d x d t \\
+s^{2} \lambda^{2} \int_{Q} \bar{\varphi}^{2} k e^{2 s \bar{\eta}}|\nabla \beta|_{\boldsymbol{\sigma}}^{2}|\bar{\psi}|^{4} d x d t+\int_{Q} k^{2} e^{4 s \bar{\eta}}|\bar{\psi}|^{6} d x d t \\
\leq\left\|e^{-s \bar{\eta}} G_{1}\right\|_{2}^{2}+s^{3} \lambda^{4} \int_{Q} \bar{\varphi}^{3}|\bar{\psi}|^{2} d x d t+s \lambda \int_{Q} \bar{\varphi}|\nabla \bar{\psi}|^{2} d x d t \\
+s \lambda^{2} \int_{Q} e^{2 s \bar{\eta}} \bar{\varphi}^{2}|\bar{\psi}|^{4} d x d t+\int_{Q} e^{4 s \bar{\eta}}|\bar{\psi}|^{6} d x d t+X_{10} .
\end{aligned}
$$

with

$$
\begin{aligned}
X_{10} & =s^{2} \lambda \int_{\Sigma} \partial_{t} \overline{\eta \varphi}|\bar{\psi}|^{2} \boldsymbol{\sigma} \nabla \beta \cdot \nu d \Sigma-2 s \lambda^{2} \int_{\Sigma} \bar{\varphi}|\nabla \beta|_{\boldsymbol{\sigma}}^{2} \bar{\psi} \boldsymbol{\sigma} \nabla \bar{\psi} \cdot \nu d \Sigma \\
& -\int_{\Sigma} \partial_{t} \bar{\psi} \boldsymbol{\sigma} \nabla \bar{\psi} \cdot \nu d \Sigma+s \lambda \int_{\Sigma} \bar{\varphi}|\nabla \bar{\psi}|_{\boldsymbol{\sigma}}^{2} \boldsymbol{\sigma} \nabla \beta . \nu d \Sigma \\
& -\frac{1}{4} \int_{\Sigma} e^{2 s \bar{\eta}} k \bar{\psi}^{3} \boldsymbol{\sigma} \nabla \bar{\psi} \cdot \nu d \Sigma+\frac{1}{4} s \lambda \int_{\Sigma} e^{2 s \bar{\eta}} k \bar{\varphi}|\bar{\psi}|^{4} \boldsymbol{\sigma} \nabla \beta . \nu d \Sigma \\
& -s^{3} \lambda^{3} \int_{\Sigma} \bar{\varphi}^{3}|\nabla \beta|_{\boldsymbol{\sigma}}^{2}|\bar{\psi}|^{2} \boldsymbol{\sigma} \nabla \beta . \nu d \Sigma-2 s \lambda \int_{\Sigma} \bar{\varphi}|\boldsymbol{\sigma} \nabla \bar{\psi} \cdot \nu|^{2} \boldsymbol{\sigma} \nabla \beta . \nu d \Sigma .
\end{aligned}
$$

Remark 2. First, we notice that $X_{10}=-X_{9}$. From the definitions of $\varphi, \bar{\varphi}, \eta$ and $\bar{\eta}$ we have

$$
\bar{\varphi} \leq \varphi, \quad \eta \leq \bar{\eta}, \quad \text { and } \quad|\bar{\psi}| \leq|\psi|, \quad \text { in } \quad Q .
$$

Additionally, since we have $\bar{\psi}=e^{-s(\bar{\eta}-\eta)} \psi$ then, we obtain

$$
|\nabla \bar{\psi}| \leq C(|\nabla \psi|+s \lambda \varphi|\nabla \beta||\bar{\psi}|) \leq C(|\nabla \psi|+s \lambda \varphi|\psi|),
$$

where the constant $c$ depends only on $\beta$.

By summing equations (75) and (82) and using the inequalities in Remark 2, we prove Lemma 3.3. 
Stability results of the monodomain system

Step 4. Using the fundamental properties of the function $\beta$ given by Lemma 3.1, we have the following result.

\section{Lemma 3.4.}

$$
\begin{aligned}
\left\|L_{1}(\psi)\right\|_{2}^{2}+\| & L_{2}(\psi) \|_{2}^{2}+s^{3} \lambda^{4} \int_{Q} \varphi^{3}|\psi|^{2} d x d t \\
& +s \lambda^{2} \int_{Q} \varphi|\nabla \psi|^{2} d x d t+s^{2} \lambda^{2} \int_{Q} \varphi^{2} e^{2 s \eta}|\psi|^{4}+\int_{Q} e^{4 s \eta}|\psi|^{6} d x d t \\
& \leq 2\left\|e^{-s \eta} G_{1}\right\|_{2}^{2}+C\left(s^{3} \lambda^{4} \int_{Q_{\omega_{0}}} \varphi^{3}|\psi|^{2} d x d t\right. \\
& \left.+s \lambda^{2} \int_{Q_{\omega_{0}}} \varphi|\nabla \psi|^{2} d x d t+s^{2} \lambda^{2} \int_{Q_{\omega_{0}}} \varphi^{2} e^{2 s \eta}|\psi|^{4} d x d t\right) .
\end{aligned}
$$

Proof. From Lemma 3.1, we see that $|\nabla \beta|$ has a lower bound on $\Omega \backslash \omega_{0}$ and therefore on $Q \backslash Q_{\omega_{0}}$ (note that $\beta$ does not depend on $t$ ), then there exists a constant $\delta>0$ such that $|\nabla \beta| \geq \delta$ in $Q \backslash Q_{\omega_{0}}$, so the left-hand side terms of (82) have the following lower bounds:

$$
\begin{aligned}
s^{3} \lambda^{4} \int_{Q} \varphi^{3}|\nabla \beta|_{\boldsymbol{\sigma}}^{4}|\psi|^{2} d x d t & \geq s^{3} \lambda^{4} \int_{Q \backslash Q_{\omega_{0}}} \varphi^{3}|\nabla \beta|_{\boldsymbol{\sigma}}^{4}|\psi|^{2} d x d t \\
& \geq \alpha_{\sigma} \delta^{4} s^{3} \lambda^{4} \int_{Q \backslash Q_{\omega_{0}}} \varphi^{3}|\psi|^{2} d x d t \\
& =\alpha_{\sigma} \delta^{4}\left(s^{3} \lambda^{4} \int_{Q} \varphi^{3}|\psi|^{2} d x d t-s^{3} \lambda^{4} \int_{Q_{\omega_{0}}} \varphi^{3}|\psi|^{2} d x d t\right) .
\end{aligned}
$$

Similarly, we have

$$
\begin{aligned}
s \lambda^{2} \int_{Q} \varphi|\nabla \beta|_{\boldsymbol{\sigma}}^{2}|\nabla \psi|_{\boldsymbol{\sigma}}^{2} d x d t & \geq s \lambda^{2} \int_{Q \backslash Q_{\omega_{0}}} \varphi|\nabla \beta|_{\boldsymbol{\sigma}}^{2}|\nabla \psi|_{\boldsymbol{\sigma}}^{2} d x d t \\
& \geq \alpha_{\sigma^{2}} \delta^{2} s \lambda^{2} \int_{Q \backslash Q_{\omega_{0}}} \varphi|\nabla \psi|^{2} d x d t \\
& =\alpha_{\sigma^{2}} \delta^{2}\left(s \lambda^{2} \int_{Q} \varphi|\nabla \psi|^{2} d x d t-s \lambda^{2} \int_{Q_{\omega_{0}}} \varphi|\nabla \psi|^{2} d x d t\right)
\end{aligned}
$$

and

$$
\begin{aligned}
s^{2} \lambda^{2} \int_{Q} \varphi^{2} k e^{2 s \eta} & |\nabla \beta|_{\boldsymbol{\sigma}}^{2}|\psi|^{4} d x d t \geq s^{2} \lambda^{2} \int_{Q \backslash Q_{\omega_{0}}} \varphi^{2} k e^{2 s \eta}|\nabla \beta|_{\boldsymbol{\sigma}}^{2}|\psi|^{4} d x d t \\
& \geq c_{k} \alpha_{\sigma} \delta^{2} s^{2} \lambda^{2} \int_{Q \backslash Q_{\omega_{0}}} \varphi^{2} e^{2 s \eta}|\psi|^{4} d x d t \\
& =c_{k} \alpha_{\sigma} \delta^{2}\left(s^{2} \lambda^{2} \int_{Q} \varphi^{2} e^{2 s \eta}|\psi|^{4} d x d t-s^{2} \lambda^{2} \int_{Q_{\omega_{0}}} \varphi^{2} e^{2 s \eta}|\psi|^{4} d x d t\right),
\end{aligned}
$$

where $c_{\boldsymbol{\sigma}}$ (respectively $c_{\boldsymbol{\sigma}^{2}}$ ) is the coercivity coefficient of $\boldsymbol{\sigma}$ (respectively $\boldsymbol{\sigma}^{2}$ ). 
Stability results of the monodomain system

Making use of these estimates (88)-(90) in (82), we have

$$
\begin{aligned}
\left\|L_{1}(\psi)\right\|^{2}+\| & L_{2}(\psi) \|^{2}+\alpha_{\sigma} \delta^{4} s^{3} \lambda^{4} \int_{Q} \varphi^{3}|\psi|^{2} d x d t+\alpha_{\sigma^{2}} \delta^{2} s \lambda^{2} \int_{Q} \varphi|\nabla \psi|^{2} d x d t \\
& +c_{k} \alpha_{\sigma} \delta^{2} s^{2} \lambda^{2} \int_{Q} \varphi^{2} e^{2 s \eta}|\psi|^{4}+c_{k^{2}} \int_{Q} e^{4 s \eta}|\psi|^{6} d x d t \\
& \leq 2\left\|e^{-s \eta} G_{1}\right\|_{2}^{2}+C\left(s^{2} \lambda^{4} \int_{Q} \varphi^{3}|\psi|^{2} d x d t+s \lambda \int_{Q} \varphi|\nabla \psi|^{2} d x d t\right. \\
& \left.+s \lambda^{2} \int_{Q} e^{2 s \eta} \varphi^{2}|\psi|^{4} d x d t\right) \\
& +\alpha_{\sigma} \delta^{4} s^{3} \lambda^{4} \int_{Q_{\omega_{0}}} \varphi^{3}|\psi|^{2} d x d t+\alpha_{\sigma^{2}} \delta^{2} s \lambda^{2} \int_{Q_{\omega_{0}}} \varphi|\nabla \psi|^{2} d x d t \\
& +c_{k} \alpha_{\sigma} \delta^{2} s^{2} \lambda^{2} \int_{Q_{\omega_{0}}} \varphi^{2} e^{2 s \eta}|\psi|^{4} d x d t .
\end{aligned}
$$

Now we note that for any $s$ sufficiently large such that $s>s_{0}=2 \max \left(\frac{C}{\alpha_{\sigma} \delta^{4}}, \frac{C}{\alpha_{\sigma} \delta^{2} c_{k}}\right)$ and for any $\lambda$ such that $\lambda>\lambda_{0}=2 \frac{C}{\alpha_{\sigma^{2}} \delta^{2}}$, the all integral terms on $Q$ and can be absorbed. Then we have

$$
\begin{aligned}
\left\|L_{1}(\psi)\right\|^{2}+\| & L_{2}(\psi) \|^{2}+s^{3} \lambda^{4} \int_{Q} \varphi^{3}|\psi|^{2} d x d t+s \lambda^{2} \int_{Q} \varphi|\nabla \psi|^{2} d x d t \\
& +s^{2} \lambda^{2} \int_{Q} \varphi^{2} e^{2 s \eta}|\psi|^{4} d x d t+\int_{Q} e^{4 s \eta}|\psi|^{6} d x d t \\
& \leq C\left(\left\|e^{-s \eta} G_{1}\right\|_{2}^{2}+s^{3} \lambda^{4} \int_{Q_{\omega_{0}}} \varphi^{3}|\psi|^{2} d x d t+s \lambda^{2} \int_{Q_{\omega_{0}}} \varphi|\nabla \psi|^{2} d x d t\right. \\
& \left.+s^{2} \lambda^{2} \int_{Q_{\omega_{0}}} \varphi^{2} e^{2 s \eta}|\psi|^{4} d x d t\right) .
\end{aligned}
$$

Step 5. Back to the original variable $V$.

\section{Lemma 3.5.}

$$
\begin{aligned}
\left\|L_{1}(\psi)\right\|_{2}^{2}+\| & L_{2}(\psi) \|_{2}^{2}+s^{3} \lambda^{4} \int_{Q} e^{-2 s \eta} \varphi^{3}|V|^{2} d x d t \\
& +s \lambda^{2} \int_{Q} e^{2 s \eta} \varphi|\nabla V|^{2} d x d t+s^{2} \lambda^{2} \int_{Q} e^{-2 s \eta} \varphi^{2}|V|^{4} d x d t+\int_{Q} e^{-2 s \eta}|V|^{6} d x d t \\
& \leq C\left(\left\|e^{-s \eta} G_{1}\right\|_{2}^{2}+s^{3} \lambda^{4} \int_{Q_{\omega_{0}}} e^{-2 s \eta} \varphi^{3}|V|^{2} d x d t\right. \\
& \left.+s \lambda^{2} \int_{Q_{\omega_{0}}} e^{-2 s \eta} \varphi|\nabla V|^{2} d x d t+s^{2} \lambda^{2} \int_{Q_{\omega_{0}}} \varphi^{2} e^{-2 s \eta}|V|^{4} d x d t\right)
\end{aligned}
$$


Stability results of the monodomain system

Proof. Recall that $\psi=e^{-s \eta} V$. Then,

$$
\nabla \psi=e^{-s \eta}(\nabla V-s \nabla \eta V)=e^{-s \eta}(\nabla V+s \lambda \varphi \nabla \beta V),
$$

and its implies

$$
\begin{aligned}
& s \lambda^{2} \int_{Q_{\omega_{0}}} \varphi|\nabla \psi|^{2} d x d t \\
& \quad \leq C\left(s^{3} \lambda^{4} \int_{Q_{\omega_{0}}} \varphi^{3} e^{-2 s \eta}|V|^{2} d x d t+s \lambda^{2} \int_{Q_{\omega_{0}}} \varphi e^{-2 s \eta}|\nabla V|^{2} d x d t\right) .
\end{aligned}
$$

We also have

$$
|\nabla \psi|^{2}=e^{-2 s \eta}\left(|\nabla V|^{2}+s^{2}|\nabla \eta|^{2}|V|^{2}-2 s V \nabla \eta . \nabla V\right) .
$$

Besides, we have

$$
\begin{gathered}
\left|2 s^{2} \lambda^{2} \int_{Q} e^{-2 s \eta} \varphi V \nabla \eta \cdot \nabla V\right| d x d t \leq \int_{Q}\left(2 e^{-s \eta} s^{3 / 2} \lambda \varphi^{1 / 2}|\nabla \eta||V|\right)\left(e^{-s \eta} s^{1 / 2} \lambda \varphi^{1 / 2}|\nabla V|\right) d x d t \\
\leq 2 s^{3} \lambda^{2} \int_{Q} e^{-2 s \eta} \varphi|\nabla \eta|^{2}|V|^{2} d x d t+\frac{1}{2} s \lambda^{2} \int_{Q} e^{-2 s \eta} \varphi|\nabla V|^{2} d x d t
\end{gathered}
$$

Then,

$$
\begin{aligned}
-2 s^{2} \lambda^{2} \int_{Q} e^{-2 s \eta} \varphi V \nabla \eta . \nabla V \geq & -2 s^{3} \lambda^{2} \int_{Q} e^{-2 s \eta} \varphi|\nabla \eta|^{2}|V|^{2} d x d t \\
& -\frac{1}{2} s \lambda^{2} \int_{Q} e^{-2 s \eta} \varphi|\nabla V|^{2} d x d t .
\end{aligned}
$$

Note that $\nabla \eta=\lambda \varphi \nabla \beta$ and $|\nabla \beta| \leq C(\Omega, \omega, \boldsymbol{\sigma})$. Therefore, using the inequality (87), we can write

$$
\begin{aligned}
s^{3} \lambda^{2} \int_{Q} e^{-2 s \eta} \varphi & |\nabla \eta|^{2}|V|^{2} d x d t \leq C s^{3} \lambda^{4} \int_{Q} \varphi^{3}|\psi|^{2} d x d t \\
\leq & C\left(\left\|e^{-s \eta} G_{1}\right\|_{2}^{2}+s^{3} \lambda^{4} \int_{Q_{\omega_{0}}} \varphi^{3}|\psi|^{2} d x d t\right. \\
& \left.+s \lambda^{2} \int_{Q_{\omega_{0}}} \varphi|\nabla \psi|^{2} d x d t+s^{2} \lambda^{2} \int_{Q_{\omega_{0}}} \varphi^{2} e^{2 s \eta}|\psi|^{4} d x d t\right)
\end{aligned}
$$

Using the inequality in (95), we modify (99) to find

$$
\begin{aligned}
& s^{3} \lambda^{2} \int_{Q} e^{-2 s \eta} \varphi|\nabla \eta|^{2}|V|^{2} d x d t \leq C\left(\left\|e^{-s \eta} G_{1}\right\|_{2}^{2}+s^{2} \lambda^{2} \int_{Q_{\omega_{0}}} \varphi^{2} e^{-2 s \eta}|V|^{4} d x d t\right. \\
& \left.\quad+s^{3} \lambda^{4} \int_{Q_{\omega_{0}}} \varphi^{3} e^{-2 s \eta}|V|^{2} d x d t+s \lambda^{2} \int_{Q_{\omega_{0}}} \varphi e^{-2 s \eta}|\nabla V|^{2} d x d t\right) .
\end{aligned}
$$

We conclude then Lemma 3.5.

\section{Step 6.(End of the proof of Theorem 3.1)}


Stability results of the monodomain system

Proof. From the first equation of (19), we have

$$
\begin{aligned}
\int_{Q} e^{-2 s \eta}(s \varphi)^{-1}\left(\partial_{t} V-\operatorname{div}(\boldsymbol{\sigma} \nabla V)+k V^{3}\right)^{2} d x d t & =\int_{Q} e^{-2 s \eta}(s \varphi)^{-1}\left|G_{1}\right|^{2} d x d t \\
& \leq C \int_{Q} e^{-2 s \eta}\left|G_{1}\right|^{2} d x d t .
\end{aligned}
$$

To complete the proof, we have to estimate the integral

$$
\begin{gathered}
\int_{Q} e^{-2 s \eta}(s \varphi)^{-1}\left(\left|\partial_{t} V\right|^{2}+|\operatorname{div}(\boldsymbol{\sigma} \nabla V)|^{2}+k^{2}|V|^{6}-2 \partial_{t} V \operatorname{div}(\boldsymbol{\sigma} \nabla V)\right. \\
+2 k \partial_{t} V V^{3}-2 k V^{3} \operatorname{div}(\boldsymbol{\sigma} \nabla V) d x d t .
\end{gathered}
$$

Now we will examine each term of (102).

$$
\begin{aligned}
& -2 \int_{Q} e^{-2 s \eta}(s \varphi)^{-1} \partial_{t} V d i v(\boldsymbol{\sigma} \nabla V) d x d t=2 \int_{Q} \nabla\left(e^{-2 s \eta}(s \varphi)^{-1} \partial_{t} V\right) \boldsymbol{\sigma} \nabla V d x d t \\
& \quad=2 \int_{Q} \nabla\left(e^{-2 s \eta}(s \varphi)^{-1}\right) \partial_{t} V \boldsymbol{\sigma} \nabla V d x d t+2 \int_{Q} e^{-2 s \eta}(s \varphi)^{-1} \nabla\left(\partial_{t} V\right) \boldsymbol{\sigma} \nabla V d x d t
\end{aligned}
$$

Here we have used $\boldsymbol{\sigma} \nabla V \cdot \nu=0$ on $\Sigma$.

We have

$$
\partial_{t} \nabla V . \boldsymbol{\sigma} \nabla V=\partial_{t}|\nabla V|_{\boldsymbol{\sigma}}^{2}-\frac{1}{2} \partial_{t} \sum_{i} \boldsymbol{\sigma}_{i i}\left|\partial_{i} V\right|^{2}
$$

therefore (103) becomes

$$
\begin{aligned}
& -2 \int_{Q} e^{-2 s \eta}(s \varphi)^{-1} \partial_{t} V d i v(\boldsymbol{\sigma} \nabla V) d x d t=2 \int_{Q} \nabla\left(e^{-2 s \eta}(s \varphi)^{-1}\right) \partial_{t} V \boldsymbol{\sigma} \nabla V d x d t \\
& \quad-\int_{Q} e^{-2 s \eta}(s \varphi)^{-1} \partial_{t} \sum_{i} \boldsymbol{\sigma}_{i i}\left|\partial_{i} V\right|^{2} d x d t+2 \int_{Q} e^{-2 s \eta}(s \varphi)^{-1} \partial_{t}|\nabla V|_{\boldsymbol{\sigma}}^{2} d x d t \\
& \quad=2 \int_{Q} e^{-2 s \eta}\left(2 \lambda-\lambda(s \varphi)^{-1}\right) \partial_{t} V \boldsymbol{\sigma} \nabla V \nabla \beta d x d t \\
& \quad+\int_{Q} e^{-2 s \eta}\left(-2 \partial_{t} \eta \varphi^{-1}-\partial_{t} \varphi s^{-1} \varphi^{-2}\right)\left(\sum_{i} \boldsymbol{\sigma}_{i i}\left|\partial_{i} V\right|^{2}-2|\nabla V|_{\boldsymbol{\sigma}}^{2}\right) d x d t .
\end{aligned}
$$

From (14)-(16), we have

$$
\left|-2 \partial_{t} \eta \varphi^{-1}-\partial_{t} \varphi s^{-1} \varphi^{-2}\right| \leq C s \varphi,
$$

and

$$
\left.\left|\sum_{i} \sigma_{i i}\right| \partial_{i} V\right|^{2}-\left.2|\nabla V|_{\boldsymbol{\sigma}}^{2}|\leq 3 C| \nabla V\right|^{2} .
$$

Therefore

$$
\begin{aligned}
& \int_{Q} e^{-2 s \eta}\left(-2 \partial_{t} \eta \varphi^{-1}-\partial_{t} \varphi s^{-1} \varphi^{-2}\right)\left(\sum_{i} \sigma_{i i}\left|\partial_{i} V\right|^{2}-2|\nabla V|_{\sigma}^{2}\right) d x d t \\
& \geq-C \int_{Q} e^{-2 s \eta} s \varphi|\nabla V|^{2} d x d t
\end{aligned}
$$


Stability results of the monodomain system

and using Young's inequality we have

$$
\begin{aligned}
& 2 \int_{Q} e^{-2 s \eta}\left(2 \lambda-\lambda(s \varphi)^{-1}\right) \partial_{t} V \boldsymbol{\sigma} \nabla V \nabla \beta d x d t \\
& \geq-\frac{1}{2} \int_{Q} e^{-2 s \eta}(s \varphi)^{-1}\left|\partial_{t} V\right|^{2} d x d t-C \int_{Q} e^{-2 s \eta} s \lambda^{2} \varphi|\nabla V|^{2} d x d t
\end{aligned}
$$

Substituting (108) and (109) in (105), we obtain

$$
\begin{aligned}
& -2 \int_{Q} e^{-2 s \eta}(s \varphi)^{-1} \partial_{t} V d i v(\boldsymbol{\sigma} \nabla V) d x d t \\
& \geq-\frac{1}{2} \int_{Q} e^{-2 s \eta}(s \varphi)^{-1}\left|\partial_{t} V\right|^{2} d x d t-C \int_{Q} e^{-2 s \eta} s \lambda^{2} \varphi|\nabla V|^{2} d x d t .
\end{aligned}
$$

For the fifth term in equation (102), using integrations by part on the time variable, we establish estimates for the product of $\partial_{t} V$ and $V^{3}$ as follows

$$
\begin{array}{rl}
2 \int_{Q} e^{-2 s \eta}(s \varphi)^{-1} & k \partial_{t} V V^{3} d x d t=-\frac{1}{2} \int_{Q} s^{-1} \partial_{t}\left(e^{-2 s \eta}(s \varphi)^{-1} k\right)|V|^{4} d x d t \\
& =-\frac{1}{2} \int_{Q} e^{-2 s \eta}\left(-2 s \partial_{t} \eta \varphi^{-1} k+\partial_{t} k \varphi^{-1}-\varphi^{-2} \partial_{t} \varphi\right)|V|^{4} d x d t .
\end{array}
$$

As consequence from (15) and (16), we have

$$
\left|-2 \partial_{t} \eta \varphi^{-1} k+s^{-1} \partial_{t} k \varphi^{-1}-s^{-1} \varphi^{-2} \partial_{t} \varphi\right| \leq C s^{2} \varphi^{2},
$$

and then

$$
2 \int_{Q} e^{-2 s \eta}(s \varphi)^{-1} k \partial_{t} V V^{3} d x d t \geq-c s^{2} \int_{Q} \varphi^{2} e^{-2 s \eta}|V|^{4} d x d t .
$$

Let us now estimate the product of the term $V^{3}$ and $\operatorname{div}(\boldsymbol{\sigma} \nabla V)$ with the integral term. Applying the Green's formula and observing that $\sigma \nabla V . \nu=0$ on $\Sigma$, we get

$$
\begin{aligned}
-2 \int_{Q} e^{-2 s \eta} k( & s \varphi)^{-1} V^{3} d i v(\boldsymbol{\sigma} \nabla V) d x d t \\
& =2 \int_{Q} \nabla\left(e^{-2 s \eta} k(s \varphi)^{-1} V^{3}\right) \boldsymbol{\sigma} \nabla V d x d t-2 \int_{\Sigma} e^{-2 s \eta} k(s \varphi)^{-1} V^{3} \boldsymbol{\sigma} \nabla V . \nu d \Sigma \\
& =2 \int_{Q} e^{-2 s \eta} s^{-1} \nabla k \varphi^{-1} V^{3} \boldsymbol{\sigma} \nabla V d x d t+4 \lambda \int_{Q} e^{-2 s \eta} k V^{3} \boldsymbol{\sigma} \nabla V . \nabla \beta d x d t \\
& -2 s^{-1} \lambda \int_{Q} e^{-2 s \eta} k \varphi^{-1} V^{3} \boldsymbol{\sigma} \nabla V . \nabla \beta d x d t+6 s^{-1} \int_{Q} e^{-2 s \eta} k \varphi^{-1} V^{2}|\nabla V|_{\boldsymbol{\sigma}}^{2} d x d t \\
& =p_{1}+p_{2}+p_{3}+p_{4} .
\end{aligned}
$$

where $p_{i}, i=1, \cdots, 4$ correspond to the $i^{\text {th }}$ term in the right hand side of (114) Taking account

$$
c \leq \varphi \leq \frac{T^{4}}{16} \varphi^{3}, \quad \varphi^{-2} \leq \frac{T^{4}}{16 c} \varphi,
$$


Stability results of the monodomain system

we can estimate $p_{1}$ as follows

$$
\begin{aligned}
\left|p_{1}\right| & \leq C \int_{Q} s^{-1} e^{-2 s \eta} \varphi^{-1}|V|^{3}|\nabla V| d x d t \\
& =C s^{-1} \int_{Q}\left(e^{-s \eta}|V|^{3} s^{1 / 2}\right)\left(e^{-s \eta} s^{1 / 2} \varphi^{-1}|\nabla V|\right) d x d t \\
& \leq C\left(\int_{Q} e^{-2 s \eta}|V|^{6} d x d t+\int_{Q} e^{-2 s \eta} \varphi^{-2}|\nabla V|^{2} d x d t\right) \\
& \leq C\left(\int_{Q} e^{-2 s \eta}|V|^{6} d x d t+s \int_{Q} e^{-2 s \eta} \varphi|\nabla V|^{2} d x d t\right) .
\end{aligned}
$$

Thus we obtain

$$
\left|p_{1}\right| \leq e^{-2 s \eta}|V|^{6} d x d t+s \int_{Q} e^{-2 s \eta} \varphi|\nabla V|^{2} d x d t .
$$

We repeat the calculations as explained in the above for $p_{2}$ and $p_{3}$, we conclude that

$$
\left|p_{1}\right|+\left|p_{2}\right|+\left|p_{3}\right| \leq C\left(\int_{Q} e^{-2 s \eta}|V|^{6} d x d t+s \int_{Q} e^{-2 s \eta} \varphi|\nabla V|^{2} d x d t\right) .
$$

Hence

$$
\begin{aligned}
-2 \int_{Q} e^{-2 s \eta} & k(s \varphi)^{-1} V^{3} \operatorname{div}(\boldsymbol{\sigma} \nabla V) d x d t \\
& \geq-C\left(\int_{Q} e^{-2 s \eta}|V|^{6} d x d t+s \int_{Q} e^{-2 s \eta} \varphi|\nabla V|^{2} d x d t\right) .
\end{aligned}
$$

Here we have used $p_{4} \geq 0$.

The last term in equation (102) is treated as follows

$$
\left.\left.\left|\int_{Q} e^{-2 s \eta}(s \varphi)^{-1} k^{2}\right| V\right|^{6} d x d t\left|\leq C \int_{Q} e^{-2 s \eta}\right| V\right|^{6} d x d t .
$$

Finally making use of the estimates (110), (113), (118) and (119) we obtain

$$
\begin{aligned}
\int_{Q} e^{-2 s \eta}(s \varphi)^{-1} & \left|\partial_{t} V\right|^{2} d x d t+\int_{Q} e^{-2 s \eta}(s \varphi)^{-1}|\operatorname{div}(\boldsymbol{\sigma} \nabla V)|^{2} d x d t \\
\leq & C\left(\int_{Q} e^{-2 s \eta}\left|G_{1}\right|^{2} d x d t+s \lambda^{2} \int_{Q} e^{-2 s \eta} \varphi|\nabla V|^{2} d x d t\right. \\
& \left.s^{2} \lambda^{2} \int_{Q} \varphi^{2} e^{-2 s \eta}|V|^{4} d x d t+\int_{Q} e^{-2 s \eta}|V|^{6} d x d t\right) .
\end{aligned}
$$

The proof of Theorem 3.1 is done by combining equation (120) and Lemma 3.5.

\section{Stability estimate of parameter $\tau_{i n}$}

In this section, we establish a the main stability result and we deduce a uniqueness result for the identification problem of parameter $\tau_{i n}$. We estimate the difference between the coefficients $a$ and $\widetilde{a}$ with an upper bound given by some Sobolev norms of the difference 
between the solutions $v_{m}$, and $\widetilde{v}_{m}$ of (17) and (18). Recall that $V=v_{m}-\widetilde{v}_{m}, W=w-\widetilde{w}$ and $q=a-\widetilde{a}$, where $a=1 / \tau_{i n}, \widetilde{a}=1 / \widetilde{\tau}_{\text {in }}$ and

$$
\begin{cases}\partial_{t} V-\operatorname{div}(\boldsymbol{\sigma} \nabla V)+k V^{3}=G_{1} & \text { in } \Omega \times(0, T) \\ \partial_{t} W=G_{2} & \text { in } \Omega \times(0, T) \\ \boldsymbol{\sigma} \nabla V \cdot \nu=0 & \text { on } \Sigma \\ V(x, 0)=V_{0}(x), \quad W(x, 0)=W_{0}(x) & \text { in } \Omega .\end{cases}
$$

The Carleman estimate (21) proved in the previous section will be the key ingredient in the proof of such a stability estimate.

For a fixed time $t_{0} \in(0, T / 2]$, let define $T_{0}=2 t_{0} \leq T$ and $\eta=\left(t\left(T_{0}-t\right)\right)^{-1}\left(e^{2 \lambda\|\beta\|_{\infty}-}\right.$ $\left.e^{\lambda \beta}\right)$. The wight function $\eta$ reaches its minimum at $t=T_{0} / 2$. We have thus obtained the following stability result.

Theorem 4.1. Let $\omega$ be a subdomain of an open set $\Omega$ of $\mathbb{R}^{3}$. Furthermore, we assume that $\widetilde{v}_{0} \in H^{2}(\Omega), \widetilde{w}_{0} \in L^{\infty}(\Omega)$ and there exists $t_{0} \in(0, T)$ such that

$$
H\left(\widetilde{v}_{m}\left(x, t_{0}\right), \widetilde{w}\left(x, t_{0}\right)\right) \geq r_{0}>0 \quad \forall x \in \Omega .
$$

Then there exists a constant $C$

$$
C=C\left(\Omega, \omega, T, r_{0}\right)>0
$$

such that

$$
\begin{aligned}
|q|^{2} \leq C\left(\|V\|_{H^{1}\left(0, T ; H^{1}\left(\omega_{0}\right)\right)}^{2}+\|V\|_{L^{4}\left(0, T ; L^{4}\left(\omega_{0}\right)\right)}^{4}+\left\|V\left(., t_{0}\right)\right\|_{H^{2}(\Omega)}^{2}\right. & \\
& \left.+\left\|V\left(., t_{0}\right)\right\|_{L^{4}(\Omega)}^{4}+\left\|V\left(., t_{0}\right)\right\|_{L^{6}(\Omega)}^{6}+\left\|W\left(., t_{0}\right)\right\|_{L^{2}(\Omega)}^{2}\right) .
\end{aligned}
$$

To prove Theorem 4.1, we need the following fundamental lemma.

Lemma 4.1. Recall that $W=w-\widetilde{w}$ where $w$ and $\widetilde{w}$ verify (17) and (18) respectively. For $t_{0}=T_{0} / 2$ and $s>0$, we have

$$
\begin{aligned}
& \int_{Q^{\prime}} e^{-2 s \eta(x, t)}|W(x, t)|^{2} d x d t \leq C\left(\int_{Q^{\prime}} e^{-2 s \eta(x, t)}\left|W\left(x, t_{0}\right)\right|^{2} d x d t+\frac{1}{s} \int_{Q^{\prime}} e^{-2 s \eta(x, t)}\left|\partial_{t} W(x, t)\right|^{2} d x d t\right) . \\
& \text { where } Q^{\prime}:=\Omega \times\left(0, T_{0}\right) .
\end{aligned}
$$

Proof. The proof is similar to [4] and [28]. From (19) we have $G_{2}=\partial_{t} W$. By the Cauchy-Schwarz inequality, we obtain

$$
\int_{Q^{\prime}}\left|\int_{t_{0}}^{t} G_{2}(x, \tau) d \tau\right|^{2} e^{-2 s \eta(x, t)} d x d t \leq \int_{Q^{\prime}}\left(\int_{t_{0}}^{t}\left|G_{2}(x, \tau)\right|^{2} d \tau\right)\left(t-t_{0}\right) e^{-2 s \eta(x, t)} d x d t
$$

Since

$$
\partial_{t} \eta(x, t)=\frac{2\left(t-t_{0}\right)}{t^{2}\left(T_{0}-t\right)^{2}}\left(e^{2 \lambda\|\beta\|_{\infty}}-e^{\lambda \beta}\right)
$$


then

$$
\begin{aligned}
\int_{Q^{\prime}} \mid \int_{t_{0}}^{t} G_{2}(x, & \tau)\left.d \tau\right|^{2} e^{-2 s \eta(x, t)} d x d t \\
\leq & C \int_{Q^{\prime}}\left(\int_{t_{0}}^{t}\left|G_{2}(x, \tau)\right|^{2} d \tau\right) \partial_{t} \eta(x, t) e^{-2 s \eta(x, t)} d x d t \\
& \leq-\frac{C}{2 s} \int_{Q^{\prime}}\left(\int_{t_{0}}^{t}\left|G_{2}(x, \tau)\right|^{2} d \tau\right) \partial_{t}\left(e^{-2 s \eta(x, t)}\right) d x d t
\end{aligned}
$$

By noting that $e^{-2 s \eta\left(x, T_{0}\right)}=e^{-2 s \eta(x, 0)}=0$, the integration by parts with respect to the time variable implies that the right hand side is equal to

$$
\frac{C}{2 s} \int_{Q^{\prime}}\left|G_{2}(x, t)\right|^{2} e^{-2 s \eta(x, t)} d x d t .
$$

We write

$$
|W(x, t)|^{2} \leq C\left(|W(x, t)-W(x, 0)|^{2}+|W(x, 0)|^{2}\right),
$$

thus the proof of Lemma 4.1 is completed.

Let us now prove the Theorem 4.1 .

Proof. Let $p=\partial_{t} V$ and we consider the time derivative of the first equation of the system (19)

$$
\partial_{t} p-\operatorname{div}(\boldsymbol{\sigma} \nabla p)=G
$$

where $G=-\partial_{t} k V^{3}-3 k V^{2} p+q \partial_{t} H+\partial_{t} R$, the functions $H, R$ are defined in (20).

First, we evaluate the first equation (19) at a fixed time $t_{0}$ such that $2 t_{0}=T_{0}<T$

$$
p\left(x, t_{0}\right)-\operatorname{div}\left(\boldsymbol{\sigma} \nabla V\left(x, t_{0}\right)\right)+k V^{3}\left(x, t_{0}\right)-q H\left(x, t_{0}\right)-R\left(x, t_{0}\right)=0 .
$$

Then, we integrate on $\Omega$ the square of (130) with the weight function $e^{-2 s \eta\left(x, t_{0}\right)}$, we obtain

$$
\begin{aligned}
& \int_{\Omega} e^{-2 s \eta\left(x, t_{0}\right)}|q|^{2}\left|H\left(x, t_{0}\right)\right|^{2} d x \leq \\
& C\left(\int_{\Omega} e^{-2 s \eta\left(x, t_{0}\right)}\left|p\left(x, t_{0}\right)\right|^{2} d x+\int_{\Omega} e^{-2 s \eta\left(x, t_{0}\right)}\left|\operatorname{div}\left(\boldsymbol{\sigma} \nabla V\left(x, t_{0}\right)\right)\right|^{2} d x\right. \\
& \left.\quad+\int_{\Omega} e^{-2 s \eta\left(x, t_{0}\right)}\left|V\left(x, t_{0}\right)\right|^{6} d x+\int_{\Omega} e^{-2 s \eta\left(x, t_{0}\right)}\left|R\left(x, t_{0}\right)\right|^{2} d x\right)
\end{aligned}
$$

since $k \in L^{\infty}$.

First, we notice that the fourth term of the right-hand side verify

$$
\begin{aligned}
\int_{\Omega} e^{-2 s \eta\left(x, t_{0}\right)} \mid & \left.R\left(x, t_{0}\right)\right|^{2} d x \leq C\left(\left\|e^{-s \eta\left(x, t_{0}\right)} V\left(x, t_{0}\right)\right\|_{L^{2}(\Omega)}^{2}\right. \\
& \left.+\left\|e^{-s / 2 \eta\left(x, t_{0}\right)} V\left(x, t_{0}\right)\right\|_{L^{4}(\Omega)}^{4}+\left\|e^{-s \eta\left(x, t_{0}\right)} W\left(x, t_{0}\right)\right\|_{L^{2}(\Omega)}^{2}\right) .
\end{aligned}
$$


Let us bound the first term in the right-hand side of (131)

$$
\begin{array}{r}
K_{1}=\int_{\Omega} e^{-2 s \eta\left(x, t_{0}\right)}\left|p\left(x, t_{0}\right)\right|^{2} d x=\int_{0}^{t_{0}} \int_{\Omega} \frac{d}{d t}\left(e^{-2 s \eta(x, t)}|p|^{2}\right) d x d t \\
=\int_{0}^{t_{0}} \int_{\Omega}\left(-2 s \partial_{t} \eta(x, t)|p|^{2}+2 p \partial_{t} p\right) e^{-2 s \eta(x, t)} d x d t .
\end{array}
$$

Besides, from (16) and using Young inequality, we have

$$
\begin{aligned}
\left.\left|-2 s \partial_{t} \eta(x, t)\right| p\right|^{2}+2 p \partial_{t} p \mid & \leq 2 s\left|\partial_{t} \eta(x, t)\right||p|^{2}+2|p|\left|\partial_{t} p\right| \\
& \leq C\left(s \varphi^{2}|p|^{2}+2\left(s \varphi^{1 / 2}|p|\right)\left(s^{-1} \varphi^{-1 / 2}\left|\partial_{t} p\right|\right)\right. \\
& \leq C\left(s \varphi^{2}|p|^{2}+s^{2} \varphi|p|^{2}+s^{-2} \varphi^{-1}\left|\partial_{t} p\right|^{2}\right) \\
& \leq C\left(s^{2} \varphi^{2}|p|^{2}+s^{-2} \varphi^{-1}\left|\partial_{t} p\right|^{2}\right) .
\end{aligned}
$$

Then, we apply the Carleman inequality given in Theorem 3.1 satisfied by $p$ without non linear cubic term, we obtain that for $s$ and $\lambda$

$K_{1} \leq \frac{C}{s}\left\|e^{-s \eta} G\right\|_{L^{2}\left(Q^{\prime}\right)}^{2}+C\left(s^{2} \lambda^{4} \int_{Q_{\omega_{0}}^{\prime}} e^{-2 s \eta} \varphi^{3}|p|^{2} d x d t+\lambda^{2} \int_{Q_{\omega_{0}}^{\prime}} e^{-2 s \eta} \varphi|\nabla p|^{2} d x d t\right)$,

where $Q^{\prime}=\Omega_{0} \times\left(0, T_{0}\right)$ and $Q_{\omega_{0}}^{\prime}=\omega_{0} \times\left(0, T_{0}\right)$. According to Proposition 2.1, $\widetilde{v}_{m} \in W^{1, \infty}\left(0, T_{0} ; L^{\infty}(\Omega)\right)$ and $\widetilde{w} \in W^{1, \infty}\left(0, T_{0} ; L^{\infty}(\Omega)\right)$. Then, using the definition of $G=-\partial_{t} k V^{3}-3 k V^{2} p+q \partial_{t} H+\partial_{t} R$ we obtain

$$
\begin{gathered}
\left\|e^{-s \eta} G\right\|_{L^{2}\left(Q^{\prime}\right)}^{2} \leq C\left(\int_{Q^{\prime}} e^{-2 s \eta}\left(|V|^{6}+|V|^{4}+|V|^{2}+|p|^{2}+|W|^{2}+\left|\partial_{t} W\right|^{2}\right) d x d t\right. \\
\left.+\int_{Q^{\prime}} e^{-2 s \eta}|q|^{2} d x d t\right)
\end{gathered}
$$

since $\widetilde{v}_{m} \in L^{\infty}\left(Q^{\prime}\right)$. Then, we apply again the Carleman inequality given in Theorem 3.1 satisfied by $p$ without non linear cubic term,

$$
\begin{gathered}
\int_{Q^{\prime}} e^{-2 s \eta}|p|^{2} d x d t \leq C\left(s^{-3}\left\|e^{-s \eta} G\right\|_{L^{2}\left(Q^{\prime}\right)}^{2}+\lambda^{4} \int_{Q_{\omega_{0}}^{\prime}} e^{-2 s \eta} \varphi^{3}|p|^{2} d x d t\right. \\
\left.+s^{-2} \lambda^{2} \int_{Q_{\omega_{0}}^{\prime}} e^{-2 s \eta} \varphi|\nabla p|^{2} d x d t\right) .
\end{gathered}
$$

Let us now estimate the following term $B=\int_{Q^{\prime}} e^{-2 s \eta}\left|\partial_{t} W\right|^{2} d x d t$. From system (19), we have

$$
\partial_{t} W=G_{2}=g\left(v_{m}, w\right)-g\left(\widetilde{v}_{m}, \widetilde{w}\right) .
$$

We write the Taylor-Lagrange development of the function $g$ at point $\left(\widetilde{v}_{m}, \widetilde{w}\right)$, there exists $\theta \in[0,1]$ such that

$$
\begin{aligned}
G_{2}=\sum_{i=1}^{2} & \left(\frac{V^{i}}{i !} \frac{\partial^{i} g\left(\widetilde{v}_{m}, \widetilde{w}\right)}{\partial v^{i}}+\frac{V^{i-1} W}{(i-1) !} \frac{\partial^{i} g\left(\widetilde{v}_{m}, \widetilde{w}\right)}{\partial v^{i-1} \partial w}\right) \\
& +\frac{V^{3}}{6} \frac{\partial^{3} g\left(\widetilde{v}_{m}+\theta h_{1}, \widetilde{w}+\theta h_{2}\right)}{\partial v^{3}}+\frac{V^{2} W}{2} \frac{\partial^{3} g\left(\widetilde{v}_{m}+\theta h_{1}, \widetilde{w}+\theta h_{2}\right)}{\partial v^{2} \partial w} .
\end{aligned}
$$


Stability results of the monodomain system

Now, since the function $g$ and its all partial derivatives are expressed as a function of the hyperbolic tangent function, which is uniformly bounded, then we deduce that

$$
\left|\partial_{t} W\right|^{2}=\left|G_{2}\right|^{2} \leq|V|^{6}+|V|^{4}+|V|^{2}+|W|^{2} .
$$

Substituting (137), (140) in (136), we obtain

$$
\begin{gathered}
\left(1-C s^{-3}\right)\left\|e^{-s \eta} G\right\|_{L^{2}\left(Q^{\prime}\right)}^{2} \leq C\left(\int_{Q^{\prime}} e^{-2 s \eta}\left(|V|^{6}+|V|^{4}+|V|^{2}+|W|^{2}\right) d x d t\right. \\
+s^{-2} \lambda^{2} \int_{Q_{\omega_{0}}^{\prime}} e^{-2 s \eta} \varphi|\nabla p|^{2} d x d t+\lambda^{4} \int_{Q_{\omega_{0}}^{\prime}} e^{-2 s \eta} \varphi^{3}|p|^{2} d x d t \\
\left.+\int_{Q^{\prime}} e^{-2 s \eta}|q|^{2} d x d t\right) .
\end{gathered}
$$

Using the Carleman inequality given by Theorem 3.1 for the estimation of $|V|^{6}+|V|^{4}+$ $|V|^{2}$ and using Lemma 4.1 for $|W|^{2}$, we get, for $s$ and $\lambda$ large enough

$$
\begin{gathered}
\left(1-s^{-3}\right)\left\|e^{-s \eta} G\right\|_{L^{2}\left(Q^{\prime}\right)}^{2} \leq C\left(\int_{Q^{\prime}} e^{-2 s \eta}\left|G_{1}\right|^{2} d x d t+\int_{Q^{\prime}} e^{-2 s \eta(x, t)}\left|G_{2}\right|^{2} d x d t+\left\|W\left(., t_{0}\right)\right\|_{L^{2}(\Omega)}^{2}\right. \\
+s^{3} \lambda^{4} \int_{Q_{\omega_{0}}^{\prime}} e^{-2 s \eta} \varphi^{3}|V|^{2} d x d t+s \lambda^{2} \int_{Q_{\omega_{0}}^{\prime}} e^{-2 s \eta} \varphi|\nabla V|^{2} d x d t \\
\left.+s^{2} \lambda^{2} \int_{Q_{\omega_{0}}^{\prime}} \varphi^{2} e^{-2 s \eta}|V|^{4} d x d t+\int_{Q^{\prime}} e^{-2 s \eta}|q|^{2} d x d t\right) .
\end{gathered}
$$

Setting $D:=\int_{Q^{\prime}} e^{-2 s \eta}\left|G_{1}\right|^{2}+\int_{Q^{\prime}} e^{-2 s \eta}\left|G_{2}\right|^{2} d x d t$, and observing that $G_{1}=q H\left(\widetilde{v}_{m}, \widetilde{w}\right)+$ $R(V, W)$, we obtain

$$
D \leq C \int_{Q^{\prime}} e^{-2 s \eta}|q|^{2} d x d t+\int_{Q^{\prime}} e^{-2 s \eta}\left(|V|^{4}+|V|^{2}+|W|^{2}\right) d x d t
$$

since $v_{m}, \widetilde{v}_{m}, \widetilde{w} \in L^{\infty}(Q)$ and from (140). Using again the Carleman inequality given by Theorem 3.1 to $|V|^{4}+|V|^{2}$ and using Lemma 4.1 to $|W|^{2}$, we get, for $s$ and $\lambda$ large enough

$$
\begin{aligned}
\left(1-s^{-1}\right) D \leq & C \int_{Q^{\prime}} e^{-2 s \eta}|q|^{2} d x d t+C\left(s \lambda^{4} \int_{Q_{\omega_{0}}^{\prime}} e^{-2 s \eta} \varphi^{3}|V|^{2} d x d t+\left\|W\left(., t_{0}\right)\right\|_{L^{2}(\Omega)}^{2}\right. \\
& \left.+s^{-1} \lambda^{2} \int_{Q_{\omega_{0}}^{\prime}} e^{-2 s \eta} \varphi|\nabla V|^{2} d x d t+\lambda^{2} \int_{Q_{\omega_{0}}^{\prime}} \varphi^{2} e^{-2 s \eta}|V|^{4} d x d t\right) .
\end{aligned}
$$

Substituting (144) in (142), we obtain

$$
\begin{aligned}
& \left(1-s^{-1}\right)\left(1-s^{-3}\right)\left\|e^{-s \eta} G\right\|_{L^{2}\left(Q^{\prime}\right)}^{2} \leq C\left(\int_{Q^{\prime}} e^{-2 s \eta}|q|^{2} d x d t+s^{3} \lambda^{4} \int_{Q_{\omega_{0}}^{\prime}} e^{-2 s \eta} \varphi^{3}|V|^{2} d x d t\right. \\
& \left.\quad+s \lambda^{2} \int_{Q_{\omega_{0}}^{\prime}} e^{-2 s \eta} \varphi|\nabla V|^{2} d x d t+s^{2} \lambda^{2} \int_{Q_{\omega_{0}}^{\prime}} \varphi^{2} e^{-2 s \eta}|V|^{4} d x d t+\left\|W\left(., t_{0}\right)\right\|_{L^{2}(\Omega)}^{2}\right)
\end{aligned}
$$


Now back to the estimate (135), we can write

$$
\begin{aligned}
& s\left(1-s^{-1}\right)\left(1-s^{-3}\right) K_{1} \leq C\left(\int_{Q^{\prime}} e^{-2 s \eta}|q|^{2} d x d t+s^{3} \lambda^{4} \int_{Q_{\omega_{0}}^{\prime}} e^{-2 s \eta} \varphi^{3}|V|^{2} d x d t\right. \\
& \left.+s \lambda^{2} \int_{Q_{\omega_{0}}^{\prime}} e^{-2 s \eta} \varphi|\nabla V|^{2} d x d t+s^{2} \lambda^{2} \int_{Q_{\omega_{0}}^{\prime}} \varphi^{2} e^{-2 s \eta}|V|^{4} d x d t\right) \\
& \left.+s^{3} \lambda^{4} \int_{Q_{\omega_{0}}^{\prime}} e^{-2 s \eta} \varphi^{3}|p|^{2} d x d t+s \lambda^{2} \int_{Q_{\omega_{0}}^{\prime}} e^{-2 s \eta} \varphi|\nabla p|^{2} d x d t+\left\|W\left(., t_{0}\right)\right\|_{L^{2}(\Omega)}^{2}\right) .
\end{aligned}
$$

At last, combining hypothesis (122), inequalities (132) and (146), estimate (131) becomes:

$$
\begin{gathered}
\int_{\Omega} e^{-2 s \eta\left(x, t_{0}\right)} r_{0}^{2}|q|^{2} \leq C\left(s^{3}(s-1)^{-1}\left(s^{3}-1\right)^{-1} \int_{Q^{\prime}} e^{-2 s \eta}|q|^{2} d x d t+\|V\|_{H^{1}\left(0, T ; H^{1}\left(\omega_{0}\right)\right)}^{2}\right. \\
\left.+\|V\|_{L^{4}\left(0, T ; L^{4}\left(\omega_{0}\right)\right)}^{4}+\left\|V\left(., t_{0}\right)\right\|_{H^{2}(\Omega)}^{2}+\left\|V\left(., t_{0}\right)\right\|_{L^{4}(\Omega)}^{4}+\left\|W\left(., t_{0}\right)\right\|_{L^{2}(\Omega)}^{2}\right) . \quad(147)
\end{gathered}
$$

Hence, for $s$ and $\lambda$ large enough (such that $\left.r_{0} s(s-1)\left(s^{3}-1\right)-s^{3}>0\right)$

$$
\begin{aligned}
\int_{\Omega} e^{-2 s \eta\left(x, t_{0}\right)}|q|^{2} & \leq C\left(\|V\|_{H^{1}\left(0, T ; H^{1}\left(\omega_{0}\right)\right)}^{2}+\|V\|_{L^{4}\left(0, T ; L^{4}\left(\omega_{0}\right)\right)}^{4}+\left\|V\left(., t_{0}\right)\right\|_{H^{2}(\Omega)}^{2}\right. \\
+ & \left.\left\|V\left(., t_{0}\right)\right\|_{L^{4}(\Omega)}^{4}+\left\|W\left(., t_{0}\right)\right\|_{L^{2}(\Omega)}^{2}\right) .
\end{aligned}
$$

This ends the proof of Theorem 4.1.

With Theorem 4.1 we have the follwing uniqueness result.

Corollary 4.1. Under the same assumptions as in Theorem 4.1 and if

$$
\begin{aligned}
& v_{m}=\widetilde{v}_{m} \quad \text { in } \quad \omega_{0} \times(0, T), \\
& v_{m}\left(x, t_{0}\right)=\widetilde{v}_{m}\left(x, t_{0}\right) \quad \text { in } \quad \Omega, \\
& w\left(x, t_{0}\right)=\widetilde{w}\left(x, t_{0}\right) \quad \text { in } \quad \Omega,
\end{aligned}
$$

then $\tau_{\text {in }}=\widetilde{\tau}_{\text {in }}$.

\section{Discussion and conclusions}

The Mitchell-Schaeffer ionic model coupled to the monodomain equation has been used for model personalization based on optical imaging and magnetic resonance data in [36]. It has been used also for personalizing the model to ventricular tachycardia using $\mathrm{X}$-ray/magnetic resonance imaging and non-contact mapping procedure on a patient with heart failure in [35]. The parameter $\tau_{\text {in }}$ in both studies plays an important role in estimating the velocity of the electrical wave. The monodomain equation combined with the Mitchell-Schaeffer ionic model has also been used for detecting ischemia region using an adjoint approach combined with a level set method in two dimensional and three dimensional frameworks $[1,14]$. In these works a low value of the parameter $\tau_{\text {in }}$ has been identified to be one of the major characteristics of the ischemic region. The estimation of the parameter $\tau_{i n}$ is then crucial in these applications. 
In this paper, we established a stability estimates for the parameter identification problem in cardiac electrophysiology modeling. Our concern is about the estimation of the parameter $\tau_{\text {in }}$ to which the solution is the most sensitive. The novelty of our work comes from the fact that, we established a new Carleman inequality for a reaction diffusion equation coupled to an ordinary differential equation. The Carleman inequality that we established for the ODE was fundamental in order to prove the global Carleman estimate for non linear parabolic equation coupled with an ordinary differential equation and solving the parameter stability problem.

\section{Acknowledgements}

This work has been supported by EPICARD cooperative research program, funded by INRIA international laboratory LIRIMA. The LAMSIN researcher's work is supported on a regular basis by the Tunisian Ministry of Higher Education, Scientific Research and Technology.

[1] Diego Álvarez, Felipe Alonso-Atienza, José Luis Rojo-Álvarez, Arcadi García-Alberola, and Miguel Moscoso. Shape reconstruction of cardiac ischemia from non-contact intracardiac recordings: A model study. Mathematical and Computer Modelling, 55(5):1770-1781, 2012.

[2] Dupaix C. Ammar Khodja K., BenAbdallah A. and Kostine I. Null controllability of some systems of parabolic type by one control force. ESAIM: COVC, 11:426-448, 2005.

[3] Mourad Bellassoued, O Yu Imanuvilov, and Masahiro Yamamoto. Inverse problem for determining the density and the lamé coefficients by boundary data. SIAM J. Math., 40:638-265, 2008.

[4] Assia Benabdallah, Michel Cristofol, Patricia Gaitan, and Masahiro Yamamoto. Inverse problem for a parabolic system with two components by measurements of one component. Applicable Analysis, 88(5):683-709, 2009.

[5] Muriel Boulakia, Serge Cazeau, Miguel A Fernández, Jean-Frédéric Gerbeau, and Nejib Zemzemi. Mathematical modeling of electrocardiograms: a numerical study. Annals of biomedical engineering, 38(3):1071-1097, 2010.

[6] Muriel Boulakia, Miguel A Fernández, Jean-Frédéric Gerbeau, and Nejib Zemzemi. Numerical simulation of electrocardiograms. In Modeling of Physiological Flows, pages 77-106. Springer, 2012.

[7] Muriel Boulakia, Miguel Angel Fernández, Jean-Frédéric Gerbeau, and Nejib Zemzemi. A coupled system of pdes and odes arising in electrocardiograms modeling. Applied Mathematics Research eXpress, 2008:abn002, 2008.

[8] Muriel Boulakia, Céline Grandmont, and Axel Osses. Some inverse stability results for the bistable reaction-diffusion equation using carleman inequalities. Comptes Rendus Mathematique, 347(11):619-622, 2009.

[9] Muriel Boulakia, Elisa Schenone, and J-F Gerbeau. Reduced-order modeling for cardiac electrophysiology. application to parameter identification. International Journal for Numerical Methods in Biomedical Engineering, 28(6-7):727-744, 2012.

[10] Yves Bourgault, Yves Coudiere, and Charles Pierre. Existence and uniqueness of the solution for the bidomain model used in cardiac electrophysiology. Nonlinear analysis: Real world applications, 10(1):458-482, 2009.

[11] Aleksandr L'vovich Bukhgeim. Carleman estimates for volterra operators and uniqueness of inverse problems. Non-Classical Problems of Mathematical Physics, page 5664 (in Russian), 1981.

[12] Aleksandr L'vovich Bukhgeim and Michael V Klibanov. Global uniqueness of a class of multidimensional inverse problems. Sov. Math. Dokl., 24:244-7, 1981. 
[13] Corrado Cesare, Lassoued Jamila, Mahjoub Moncef, and Néjib Zemzemi. Stability analysis of the pod reduced order method for solving the bidomain model in cardiac electrophysiology. Mathematical Biosciences, 272:81-91, 2016.

[14] Carlos E Ch, Felipe Alonso-Atienza, Nejib Zemzemi, Yves Coudi, et al. Inverse localization of ischemia in a 3d realistic geometry: A level set approach. In 2015 Computing in Cardiology Conference (CinC), pages 229-232. IEEE, 2015.

[15] John C Clements, Jukka Nenonen, PKJ Li, and B Milan Horáček. Activation dynamics in anisotropic cardiac tissue via decoupling. Annals of biomedical engineering, 32(7):984-990, 2004.

[16] Piero Colli Franzone and Luca F Pavarino. A parallel solver for reaction-diffusion systems in computational electrocardiology. Mathematical models and methods in applied sciences, 14(06):883-911, 2004.

[17] Gaitan P. Cristofol M. and Ramoul H. Inverse problems for two by two reaction-diffusion system using a carleman estimate with one observation. Inverse Problems, 11, 2006.

[18] Schenone Elisa. Reduced order models, forward and inverse problems in cardiac electrophysiology. PhD thesis, 2014.

[19] P Colli Franzone, LF Pavarino, and B Taccardi. Simulating patterns of excitation, repolarization and action potential duration with cardiac bidomain and monodomain models. Mathematical biosciences, 197(1):35-66, 2005.

[20] Andrei Vladimirovich Fursikov and O Yu Imanuvilov. Controllability of evolution equations. Seoul National University, RIM, Seoul, South Korea, 34, 1996.

[21] Andrei Vladimirovich Fursikov and O Yu Imanuvilov. Exact controllability of the navier-stokes and boussines equations. Russian Mathematical Surveys, 54(3):565-618, 1999.

[22] Kunisch Karl and Wagner Marcus. Optimal control of the bidomain system (iv): Corrected proofs of the stability and regularity theorems.

[23] Michael V Klibanov. Inverse problems in the large and carleman bounds. Differ. Eqns, 20:755-604, 1984.

[24] Michael V Klibanov. Uniqueness in the large of solutions of inverse problems for a class of differential equations. Differ. Eqns, 20:13905, 1985.

[25] Michael V Klibanov. Uniqueness of solutions of two inverse problems for a maxwellian system. USSR Comput. Math. Math. Phys., 26:6772, 1986.

[26] Michael V Klibanov. A class of inverse problems for nonlinear parabolic equation. Siberian Math. J., 27:698-708, 1987.

[27] Michael V Klibanov. Inverse problems and carleman estimates. Inverse Problems, 8:57596, 1992.

[28] Michael V Klibanov and A Timonov. Carleman estimates for coefficient inverse problems and numerical applications. Utrecht: VSP, 2004.

[29] P Klugler. Identification of a temperature dependent heat conductivity from single boundary measurements. SIAM J. Numer. Anal., 41(4):15431563, 2004.

[30] Colleen C Mitchell and David G Schaeffer. A two-current model for the dynamics of cardiac membrane. Bulletin of mathematical biology, 65(5):767-793, 2003.

[31] N V Muzylev. Uniqueness theorems for some inverse problems of heat conduction. USSR Comput. Math. Math. Phys., 20:120-34, 1980.

[32] J.C. Neu and W. Krassowska. Homogenization of syncytial tissues a comparison of monodomain and bidomain reaction-diffusion models for action potential propagation in the human heart. IEEE Transactions on Biomedical Engineering, 21(2), 1993.

[33] Micol Pennacchio, Giuseppe Savaré, and Piero Colli Franzone. Multiscale modeling for the bioelectric activity of the heart. SIAM Journal on Mathematical Analysis, 37(4):1333-1370, 2005.

[34] M Pilant and W Rundell. An inverse problem for a nonlinear parabolic equation. Commun. Part. Differ. Eqns, 11:445-57, 1986.

[35] Jatin Relan, Phani Chinchapatnam, Maxime Sermesant, Kawal Rhode, Matt Ginks, Hervé 
Delingette, C Aldo Rinaldi, Reza Razavi, and Nicholas Ayache. Coupled personalization of cardiac electrophysiology models for prediction of ischaemic ventricular tachycardia. Interface Focus, page rsfs20100041, 2011.

[36] Jatin Relan, Maxime Sermesant, Hervé Delingette, Mihaela Pop, Graham A Wright, and Nicholas Ayache. Quantitative comparison of two cardiac electrophysiology models using personalisation to optical and mr data. In 2009 IEEE International Symposium on Biomedical Imaging: From Nano to Macro, pages 1027-1030. IEEE, 2009.

[37] Simone Scacchi and Luca F Pavarino. Multilevel Schwarz and Multigrid preconditioners for the Bidomain system. Springer, 2008.

[38] Néjib Zemzemi. Etude théorique et numérique de l'activité électrique du coeur: Applications aux électrocardiogrammes. PhD thesis, Université Paris-Sud, 2009. 